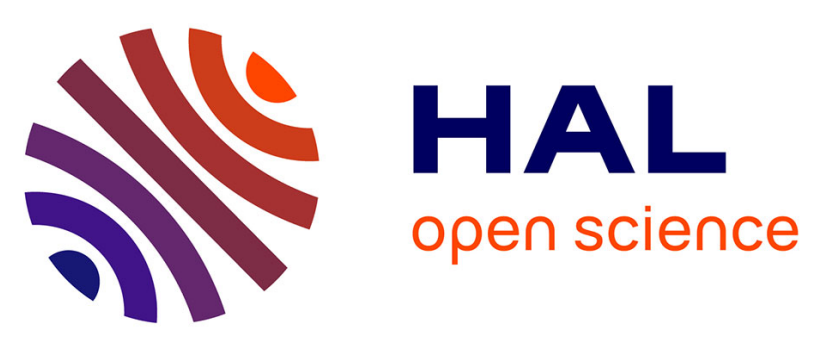

\title{
Mechanistic modeling and equilibrium prediction of the reactive extraction of organic acids with amines: a comparative study of two complexation-solvation models using 3-hydroxypropionic acid
}

Florian Chemarin, Marwen Moussa, Florent Allais, Violaine Athès, Ioan-Cristian Trelea

\section{To cite this version:}

Florian Chemarin, Marwen Moussa, Florent Allais, Violaine Athès, Ioan-Cristian Trelea. Mechanistic modeling and equilibrium prediction of the reactive extraction of organic acids with amines: a comparative study of two complexation-solvation models using 3-hydroxypropionic acid. Separation and Purification Technology, 2017, 189, pp.475-487. 10.1016/j.seppur.2017.07.083 . hal-01583081

\section{HAL Id: hal-01583081 \\ https://hal.science/hal-01583081}

Submitted on 6 Sep 2017

HAL is a multi-disciplinary open access archive for the deposit and dissemination of scientific research documents, whether they are published or not. The documents may come from teaching and research institutions in France or abroad, or from public or private research centers.
L'archive ouverte pluridisciplinaire HAL, est destinée au dépôt et à la diffusion de documents scientifiques de niveau recherche, publiés ou non, émanant des établissements d'enseignement et de recherche français ou étrangers, des laboratoires publics ou privés. 
Mechanistic modeling and equilibrium prediction of the reactive extraction of organic acids with amines: a comparative study of two complexation-solvation models using 3-hydroxypropionic acid

F. Chemarin ${ }^{a, b}$, M. Moussa ${ }^{b^{*}}$, F. Allais ${ }^{a, b}$, V. Athès ${ }^{b}$, I.C. Trelea $^{b}$

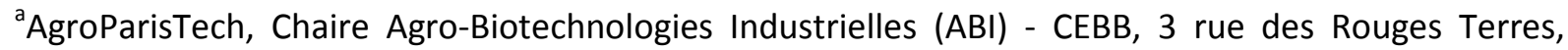
51110 Pomacle, France

${ }^{\mathrm{b}}$ UMR GMPA, AgroParisTech, INRA, Université Paris-Saclay, 78850 Thiverval-Grignon, France

* Corresponding author: marwen.moussa@inra.fr

Abstract

A comprehensive study of equilibrium states involved in the reactive extraction of 3hydroxypropionic acid (3-HP) by tri-n-octylamine (TOA) in $n$-decanol is described. Complexation phenomena between 3-HP and TOA were revealed by infrared spectroscopy (FT-IR). This study demonstrated that the main extraction mechanism is the formation of an ion pair involving the dissociated form of 3-HP. Chemical models were then formulated, taking the formation of the ion pair between 3-HP and TOA and the influence of the amount of solvent in the organic phase into account. Two models are proposed: (1) a stoichiometric model where the solvent is seen as a reagent that is involved in the complexation as a synergistic extractant, but with competition for solvation via $\mathrm{H}$-bond interactions with TOA; (2) a model where the solvent is seen as a phase modifier that improves the physicochemical properties of the extractant and that changes the complexation equilibrium. Both models describe the extraction yields in the investigated ranges, with Model (2) being particularly predictive since it provides a mean absolute prediction error of less than $2 \%$ in yield units. According to this latter model, the complexation equilibrium coefficient increases with the increase of the $n$-decanol proportion in the organic phase. Model (2) shows that solvation effects are better described as non-stoichiometric interactions. Therefore, the two mechanistic models accurately represent extraction yields over a wide range of initial conditions and are potentially 
transposable to other reactive extraction systems. Such knowledge is essential for further implementation of downstream processes.

Keywords:

Solvent extraction; organic acid; complex solvation; infrared spectroscopy; equilibrium modeling 
Abbreviations

3-HP: 3-hydroxypropionic acid

$a_{k}:$ polynomial coefficients

$A^{-}$: organic acid in dissociated form

AH: organic acid in non-dissociated form

$[A H]_{\text {ini }}$ : initial aqueous concentration of organic acid in all forms (mol/L)

c: general chemical species

C: molar concentration of a chemical species (mol/L)

$K_{11}$ : complexation equilibrium constant for a complex containing one acid molecule and one amine molecule (L/mol)

$K_{11 n}$ : complexation equilibrium constant for a 1:1 acid:amine complex including $\mathrm{n}$ solvent molecules in the complexation reaction $\left((\mathrm{L} / \mathrm{mol})^{\mathrm{n}+1}\right)$

$K_{A}$ : acidity constant of the organic acid (-)

$K_{a q}$ : equilibrium constant for the protonation of octylamine with $\mathrm{AH}(-)$

$K_{w}$ : water autoprotolysis constant (-)

m: partition coefficient of $\mathrm{AH}$ between water and solvent (-)

$\mathrm{n}$ : stoichiometric coefficient

$N_{1}$ : number of experimental values used for the calibration of the models

$\mathrm{N}_{2}$ : number of experimental values in the validation dataset

OA: octylamine

$\mathrm{OAH}^{+}$: octylammonium (protonated octylamine)

p: stoichiometric coefficient

P: number of fitted parameters in each model

RMSD: root mean squared deviation

SER: standard error of the regression

TOA: tri- $n$-octylamine 
$\mathrm{TOAH}^{+}$: trioctylammonium (protonated tri-n-octylamine)

$\mathrm{x}$ : molar fraction

z: charge of an ionic species

$|\Delta X|$ : absolute error between a model value and an experimental value for variable $X$ (unit of $X$ )

$\gamma$ : activity coefficient

$\Gamma$ : function representing non-idealities

$v$ : general stoichiometric coefficient of a chemical reaction

$\varphi$ : volume fraction of the solvent in the organic phase

$\chi$ : electronegativity (Pauling scale)

Subscripts and superscripts:

aq: in the aqueous phase

ini: initially, before extraction

mod: concerning the model predicted values

org: in the organic phase

$\mathrm{ROH}$ : concerning the solvent (in this case, $n$-decanol)

TOT: takes all the chemical speciations of a species into account 


\section{Introduction}

Reactive extraction has been widely investigated for the removal of organic acids from dilute aqueous phases, especially for organic acids produced by bioconversion. In such a process, a hydrophilic acidic molecule can be extracted based on its interaction with a basic hydrophobic amine diluted in an organic phase. Acid and amine interact with each other and are usually said to form a hydrophobic complex [1].

The scientific literature provides numerous equilibrium studies and modeling methods for the reactive extraction of organic acids with amines in different solvents. Determination of the equilibrium state and of the extraction yield from given initial conditions has been studied using several kinds of predictive and semi-predictive models. For example, the Linear Solvation Energy Relationship model that focuses on specific interactions between solutes and solvents requires a large number of tested solvents and fitted parameters [2,3]. Statistical methods based on experimental design like the response surface methodology or neural networks $[4,5]$ result in models that do not take physicochemical mechanisms into account. Thermodynamic models [6-8] consider all physicochemical interactions but require activity coefficients obtained either through complex predictive calculations or the fitting of numerous parameters for semi-predictive models, a prerequisite due to a lack of experimental data for the studied systems. However, the most frequently used model is certainly the chemical one [1,9-11] (sometimes referred to as "chemodel") thanks to its theoretical simplicity, accurate results and direct insights into extraction mechanisms. Chemical models assume that chemical interactions (i.e., specific relatively strong interactions involved in complex formation) are much stronger than physical interactions (i.e., nonspecific weak interactions such as solvation). The equilibrium behavior can be modeled by the formation of various reversible stoichiometric complexes of acids and amines with corresponding equilibrium constants. In chemodels, the stoichiometry of complexes is determined by the best fit between experimental measurements and predictions based on mass balance and mass action laws. 
Our previous study [12] highlighted strong interactions between the solvent used, i.e., decanol, and the acid-amine complex. We provided experimental results that emphasized some physicochemical interactions involved in the complex formation and the importance of the amine purity. Scientific literature reports some studies dealing with $\mathrm{C}_{3}$-monocarboxylic acid extraction using aliphatic amines in decanol with or without diluents, e.g., propionic $[13,14]$, acrylic $[15,16]$, lactic $[17,18]$ and pyruvic $[19,20]$ acids. These studies tend to show that decanol is an active diluent that interacts with acidamine complexes, increasing the yield when compared to inert diluents. In this paper, we aim at uncovering the extraction mechanisms involved in the case of 3-hydroxypropionic acid, a biobased platform molecule of industrial interest [21], with tri-n-octylamine in $n$-decanol. To confirm the complex formation and elucidate its structure, an infrared spectroscopy study was carried out. We focus here on the modeling of equilibrium states as a mechanistic and predictive tool and propose two ways of taking complexation and solvent stabilizing interactions into account over a wide range of initial conditions.

\section{Material and methods}

\subsection{Theory}

\subsubsection{Physical extraction}

When an aqueous phase of acid comes in contact with an organic phase, physical partitioning of the acid between the two phases occurs, i.e., the transfer from one phase to another due to solubility. When polar solvents such as alcohols and ketones are used, acid partitioning is reported to be independent of its concentration [1]:

$$
\left.A H_{a q} \leftrightarrow A H_{\text {org }} \quad \text { (reaction } 1\right)
$$

\subsubsection{Reactive extraction}

In an aqueous phase, acids and bases can react together through reversible reactions to give the corresponding solvated free conjugated bases and acids. In an organic phase, the weak polarity and 
dielectric constant can prevent the species from forming corresponding solvated free ions, and the acidic and basic molecules can remain bound together through acid-base interactions. These interactions can consist in $\mathrm{H}$-bondings and/or ion pair formation according to the polarity of the solvent. In this model, we assume that the acid:amine complex is an ion pair with a 1:1 stoichiometry, as suggested by the infrared spectroscopy study presented below (reaction 2):

$$
\left.A H_{a q}+\text { TOA }_{\text {org }} \leftrightarrow\left(\mathrm{TOAH}^{+}, A^{-}\right) \text {org } \quad \text { (reaction } 2\right)
$$

The validity of this assumption will be checked and further discussed in the Results, Section 3.1.

\subsubsection{Acid dissociation}

Acid dissociation in water is given by reaction 3:

$$
A H_{a q}+H_{2} O_{(l)} \leftrightarrow A_{a q}^{-}+H_{3} O_{a q}^{+} \text {(reaction 3) }
$$

The occurrence of such a reaction can reduce the amount of protonated acid in solution and has to be considered in the model since the amine extractant only reacts with the protonated form of the acid.

\subsubsection{Water autoprotolysis}

Water autoprotolysis has to be considered for acid-base equilibria in the aqueous phase (reaction 4):

$$
\left.2 \mathrm{H}_{2} \mathrm{O} \leftrightarrow \mathrm{H}_{3} \mathrm{O}_{a q}^{+}+\mathrm{HO}_{a q}^{-} \quad \text { (reaction } 4\right)
$$

\subsubsection{Amine solubility in the aqueous phase}

Our previous results [12] drew attention to the presence of impurities that reduced extraction yields for low acid concentrations. Tri-n-octylamine (TOA) impurities were identified as $n$-octylamine (OA) and di-n-octylamine. These organic impurities are extracted in the aqueous phase through reactive extraction with acidic species. Partition of long chain amines between the aqueous and the organic 
phases can be neglected but, in acidic environments, amines undergo protonation and become more soluble in water. This cannot be neglected for primary amines and is taken into account in reaction 5 :

$$
\left.A H_{a q}+O A_{\text {org }} \leftrightarrow O A H_{a q}^{+}+A_{a q}^{-} \quad \text { (reaction } 5\right)
$$

\subsection{Modeling}

\subsubsection{Model 0: Complexation without explicit solvation effects}

For the chemical system described above, three types of relationships can be used to find the equilibrium state:

\section{Ideal laws of mass action}

Although mass action laws can be expressed with different types of concentrations, we chose to use molar concentrations, which are in fact recommended for liquid solutions [22] if volumes remain constant. We empirically observed that volumes were invariant in our experiments, contrary to the total mass or number of moles. Ideal laws of mass action corresponding to reactions (1)-(5) are given by equations 1-5:

$$
\begin{gathered}
m=\frac{\left[A H_{\text {org }}\right]}{\left[A H_{a q}\right]}(1) \quad K_{11}=\frac{\left[\left(\mathrm{TOAH}^{+}, A^{-}\right)_{\text {org }}\right]}{\left[T O A_{\text {org }}\right]\left[A H_{a q}\right]}(2) \quad K_{A}=\frac{\left[A_{a q}^{-}\right]\left[H_{3} O_{a q}^{+}\right]}{\left[A H_{a q}\right]}(3) \\
K_{w}=\left[H_{3} O_{a q}^{+}\right]\left[H O_{a q}^{-}\right](4) \quad K_{a q}=\frac{\left[O A H_{a q}^{+}\right]\left[A_{a q}^{-}\right]}{\left[A H_{a q}\right]\left[O A_{\text {org }}\right]}(5)
\end{gathered}
$$

Equation (1) only holds for the decanol volume fraction of the organic phase.

\section{Material balances}

Material balances between the initial and equilibrium states are written for amine, acid and impurities (equations 6 to 8 ): 


$$
\left\{\begin{array}{c}
{[\mathrm{TOA}]_{\text {ini }}=\left[\mathrm{TOA}_{\text {org }}\right]+\left[\left(\mathrm{TOAH}^{+}, \mathrm{A}^{-}\right)_{\text {org }}\right]} \\
{[\mathrm{AH}]_{\text {ini }}=\left[\mathrm{AH} \mathrm{H}_{\text {aq }}\right]+\left[\mathrm{A}_{\text {aq }}^{-}\right]+\frac{V_{\text {org }}}{V_{\text {aq }}}\left(\left[\mathrm{AH} \mathrm{H}_{\text {org }}\right]+\left[\left(\mathrm{TOAH}^{+}, A^{-}\right)_{\text {org }}\right]\right)} \\
{\left[\mathrm{OA} A_{\text {org }}\right]_{\text {ini }}=\left[O A_{\text {org }}\right]+\frac{V_{\text {aq }}}{V_{\text {org }}}\left[O A H_{\text {aq }}^{+}\right]}
\end{array}\right.
$$

\section{$\underline{\text { 3. Electroneutrality principle }}$}

The electric charge balance is given for the ionic species involved in the considered system (equation 9):

$$
\left[A_{a q}^{-}\right]+\left[H O_{a q}^{-}\right]=\left[O A H_{a q}^{+}\right]+\left[H_{3} O_{a q}^{+}\right]
$$

All the concentrations were expressed as functions of $\left[A H_{a q}\right],\left[T O A_{\text {org }}\right]$ and $\left[O A_{\text {org }}\right]$ using equations (1) to (5) and (9), and the resulting nonlinear system with three equations and three unknowns was solved numerically. In particular, by combining equations (9) with (3)-(5), we obtain:

$$
\left[A_{a q}^{-}\right]=\sqrt{\frac{\left[A H_{a q}\right]\left(K_{a q}\left[O A_{o r g}\right]+K_{A}\right)}{1+\frac{K_{w}}{K_{A}\left[A H_{a q}\right]}}}
$$

Note that the variables are physically bounded so that there is only one set of solutions that can physically exist:

$$
\begin{gathered}
0<\left[\text { TOA } A_{\text {org }}\right]<[\text { TOA }]_{\text {ini }} \\
0<\left[A H_{\text {aq }}\right]<[A H]_{i n i} \\
0<\left[O A_{\text {org }}\right]<[O A]_{\text {ini }}
\end{gathered}
$$

Some of the reaction constants such as the acidity constant of 3-HP $\left(K_{A}=10^{-4.51}\right)$, the ionic product of water $\left(K_{w}=10^{-14}\right)$ and the partition coefficient of the acid between the aqueous and the organic phases $(m=0.02)$ are known at the experimental temperature $\left(25^{\circ} \mathrm{C}\right)$. Therefore, according to this model, there are only two equilibrium constants to be determined: $K_{11}$ and $K_{a q}$. 
Model yields and $\mathrm{pH}$ were calculated using the following equations:

$$
\begin{aligned}
& Y_{\text {mod } 0} \stackrel{\text { def }}{=} \frac{\left[A H_{\text {org,TOT }}\right]}{[A H]_{\text {ini }}}=\frac{V_{\text {org }}}{V_{a q}} \times \frac{\varphi m\left[A H_{a q}\right]+K_{11}\left[A H_{a q}\right]\left[\text { TOA } A_{\text {org }}\right]}{[A H]_{\text {ini }}} \\
& p H_{\text {mod }}=p K_{A}+\log \left(\frac{\left[A_{a q}^{-}\right]}{\left[A H_{a q}\right]}\right)
\end{aligned}
$$

where $\varphi$ is the volume fraction of the solvent in the organic phase:

$$
\varphi=\frac{V_{R O H}}{V_{\text {org }}}
$$

\subsubsection{Model 1: The solvent as a synergistic extractant}

In a previous publication [12], we showed the major role of the solvent on 3-HP extraction. We found that hydrogen-bonding ability for alcohols may be the leading mechanism of acid-amine complex stabilization in the organic phase, therefore determining the extraction yield. If complexsolvent interactions are strong enough, it is possible to consider including the solvent in the complex with a mass action law as a synergistic extractant. Alcohols are polar and protic, which gives them the considerable ability to solvate the complex resulting from the interactions between 3-HP and amines. Consequently, they have a huge importance in the extraction process. A simple way of including the solvent effect is to consider that it takes part in the reaction and to preserve the mathematical formalism of ideal solutions. To take the effects of the solvent on the extraction process into account in the case of strong interactions, Tamada et al. [9] suggested a stoichiometric reaction that includes the solvent in the complex formation as a synergistic extractant with amines:

$$
A H_{a q}+\text { TOA }_{\text {org }}+n R O H \leftrightarrow\left[\left(\mathrm{TOAH}^{+}, A^{-}\right)(\mathrm{ROH})_{n}\right]_{\text {org }} \text { (reaction 6) }
$$

This is equivalent to a synergistic reactive extraction by two extractants found in the literature for organic acids. For example, it was shown that tri- $n$-butylphosphate, a phosphorous bonded oxygen donor and a strongly solvating agent [1], was a good synergistic extractant with TOA for organic acids 
$[23,24]$, as was di-2-ethylhexylphosphoric acid $[25,26]$, a water insoluble phosphoric acid. These binary extractant systems allow stoichiometrically defined complexes consisting of three types of units: the organic acid, the amine and the synergistic extractant. The synergistic coefficient defined as the ratio between the distribution coefficient with the binary extractant and the sum of distribution coefficients of single extractants is sometimes provided to characterize the degree of synergy $[20,27]$.

Given the $\mathrm{H}$-bond acceptor character of the amine and the $\mathrm{H}$-bond donor property of the alcohol solvent, we decided to introduce competition for solvation in the organic phase according to reaction 7. This enables a higher degree of freedom for the model and an easier comparison with Model 2:

$$
T O A_{\text {org }}+p R O H \leftrightarrow\left[T O A(R O H)_{p}\right]_{\text {org }}(\text { reaction } 7)
$$

These simple assumptions reflect the loss of activity of the solvent with the increase of amine concentration, resulting in low extraction yields when the amount of solvent in the organic phase is reduced. According to this model, the stoichiometric coefficients of the solvent and the equilibrium constants of reactions 6 and 7 are the four parameters that describe the role of the solvent in the extraction mechanisms.

The ideal laws of mass action used are (1)-(5) and the ones corresponding to reaction 6 (equation (17)) and 7 (equation (18)) are:

$$
\begin{gathered}
K_{11 n}=\frac{\left[\left[\left(\mathrm{TOAH}^{+}, \mathrm{A}^{-}\right)(\mathrm{ROH})_{n}\right]_{\text {org }}\right]}{\left[\mathrm{AH}_{\text {aq }}\right]\left[\mathrm{TOA}_{\text {org }}\right][\mathrm{ROH}]^{n}} \\
K_{\text {comp }}=\frac{\left[\left[\mathrm{TOA}(\mathrm{ROH})_{p}\right]_{\text {org }}\right]}{\left[\mathrm{TOA}_{\text {org }}\right][\mathrm{ROH}]^{p}}
\end{gathered}
$$

Material balances used in this model are equations (8) and (19) to (21): 


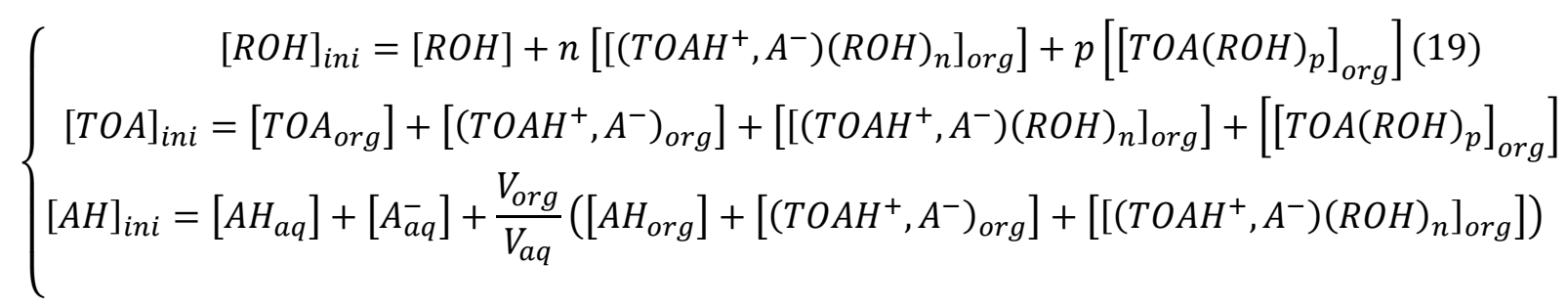

The electroneutrality principle is still given by equation (9).

All concentrations were expressed as functions of $\left[A H_{a q}\right],\left[R_{3} N_{\text {org }}\right],[R O H]$ and $\left[O A_{\text {org }}\right]$, and the resulting nonlinear system with four equations and four unknowns was solved numerically.

The physical bounds of this system are given by equations (11) to (13) and (22):

$$
0<[R O H]<[R O H]_{\text {ini }}
$$

According to this model, there are four equilibrium constants $\left(K_{11}, K_{11 n}, K_{a q}, K_{c o m p}\right)$ and two stoichiometric coefficients ( $n$ and $p$ ) to be determined.

pH was calculated according to equation (15) and the extraction yield predicted by the model using equation (23):

$$
Y_{\text {mod } 1} \stackrel{\text { def }}{=} \frac{\left[A H_{\text {org,TOT }}\right]}{[A H]_{\text {ini }}}=\frac{V_{\text {org }}}{V_{a q}} \times \frac{\varphi m\left[A H_{a q}\right]+K_{11}\left[A H_{a q}\right]\left[T O A_{\text {org }}\right]+K_{11 n}\left[A H_{a q}\right]\left[T O A_{\text {org }}\right][R O H]^{n}}{[A H]_{\text {ini }}}
$$

\subsubsection{Model 2: Composition-dependent equilibrium}

It can be shown that the law of mass action for a chemical equilibrium $\left(\sum_{i} v_{i} c_{i}=0\right)$ of a non-ideal liquid system can be expressed in the following form [22]:

$$
\sum_{i} \ln \left(C_{i}^{v_{i}}\right)=\ln \left(K_{11}^{i d}\right)+\Gamma
$$

where $\Gamma$ is a function representing non-idealities (volume effects, molecule properties and interactions, etc.) and depending on the composition of the system at constant temperature and pressure. 
In the usual representation of non-ideal behavior with molar activity coefficients, $\Gamma$ would represent the ratio of reactant activity coefficients and product activity coefficients. They only depend on the system composition at constant temperature and pressure.

$$
\Gamma=-\sum_{i} \ln \left(\gamma_{i}^{v_{i}}\right)
$$

In the aqueous phase, the main solute is the non-dissociated form of the acid that represents less than $1 \% \mathrm{~mol} / \mathrm{mol}$ in the entire tested range. In such dilute conditions, the variation of acid concentration should not significantly impact the properties of the mixture and its impact can be averaged using a constant term, assuming a constant value of the activity coefficient. In the studied range, it was shown that the variation of the activity coefficient was less than $5 \%$ for the nondissociated form of acetic acid in water, for example [28]. This contribution was thus assumed to be constant and was averaged in the equilibrium constant. In the case of diluted ions, activity coefficients can be estimated using the Davies equation [29]:

$$
\log \left(\gamma_{i}\right)=-0.5 z_{i}^{2}\left(\frac{\sqrt{I}}{1+\sqrt{I}}-0.30 I\right)(26)
$$

where $\mathrm{I}$ is the ionic strength, which is equal to the concentration of the $\mathrm{H}^{+}$ion when the acid dissociation reaction is the only source of ions in solution. Given the equilibrium $\mathrm{pH}$ of our experiments, the Davies equation gave activity coefficients of ions in solution of between 0.99 and 0.95 , varying in the same direction as the non-dissociated acid. Its impact on dissociation equilibrium calculations was therefore not taken into account. We thus assumed that all the activity coefficients in the aqueous phase were constants included in equilibrium constants, so that ideal laws of mass action were used for aqueous reactions (3) and (4). These simplifications were also made for reaction 1 since the linearity between $\left[\mathrm{AH}_{\mathrm{aq}}\right]$ and $\left[\mathrm{AH}_{\mathrm{org}}\right]$ had been previously checked over our entire concentration range [12]. They also apply to reaction 5, which is expected to have a small impact on the results when purified reactants are used. 
In the organic phase, the acid-amine complex is also rather diluted $(<5 \% \mathrm{~mol} / \mathrm{mol})$ in our case, so it could be considered, at first glance, that the physicochemical properties of the medium are mainly based on solvent, TOA and soluble water, the latter accounting for up to $28 \%$ of the total number of moles [12]. However, such a ternary system has only one degree of freedom related to its composition because the initial amount of solvent determines the amount of TOA for a given ratio, which in turn determines water content through its solubility. We can thus assume that nonidealities depend only on one variable, the initial solvent mole fraction (or, equivalently, the TOA mole fraction), and are expressed by the function:

$$
\Gamma=\Gamma\left(x_{R O H}^{i n i}\right)
$$

For convenience, this dependence was included in the equilibrium coefficient:

$$
K_{11}\left(x_{R O H}^{i n i}\right)=K_{11}^{i d} \times e^{\Gamma\left(x_{R O H}^{i n i}\right)}=\frac{\left[\left(T O A H^{+}, A^{-}\right)_{a q}\right]}{\left[A H_{a q}\right]\left[T O A_{\text {org }}\right]}
$$

According to the Stone-Weierstrass theorem, this dependence can be approximated by a polynomial function of $x_{\mathrm{ROH}}$ between $\mathrm{x}_{\mathrm{ROH}}=0$ and $\mathrm{x}_{\mathrm{ROH}}=1$ :

$$
\ln \left(K_{11}\right)=\sum_{k=0}^{m} a_{k} \cdot\left(x_{R O H}^{i n i}\right)^{k}
$$

$K_{11}\left(x_{R O H}^{i n i}\right)$ is an apparent equilibrium coefficient, which is truly constant only for a given initial molar fraction of solvent in the organic phase. This is why it is called an equilibrium coefficient and not an equilibrium constant.

This model considers the decanol/TOA mixture as a more or less favorable medium for the complexation reaction rather than viewing the solvent as a reactant of the complexation. The quantity of solvent is then unchanged over the course of the reaction. Non-idealities are taken into account in the variation of the apparent equilibrium coefficient $K_{11}$, the material balances are given by equations (6)-(8), and the electroneutrality principle by equation (9). All the concentrations were 
expressed as functions of $\left[A H_{a q}\right],\left[\mathrm{TOA}_{\text {org }}\right]$ and $\left[O A_{\text {org }}\right]$, and the resulting nonlinear system with three equations and three unknowns was solved numerically. The physical bounds of this system are (11)-(13). The parameters to be determined for this model are $K_{a q}$ and the polynomial coefficients $\left(a_{k}\right)$ of $K_{11}\left(x_{R O H}^{i n i}\right)$ in equation (27). The polynomial order was iteratively increased until the $95 \%$ confidence interval around the leading coefficient includes 0 , meaning that this coefficient was not significantly different from 0 .

$\mathrm{pH}$ was calculated according to (15) and model yields were calculated using equation (14) with $K_{11}$ given by equation (29).

\subsection{Experiments}

\subsubsection{Spectroscopic study}

Fourier Transformed Infrared (FT-IR) analyses were performed using a Cary 630 Fourier Transform InfraRed spectrometer from Agilent Technologies (California, USA). A typical organic phase made of $20 \% \mathrm{v} / \mathrm{v}$ TOA in decanol was considered as the reference. Similar organic phases were loaded with four different concentrations of 3-HP through a liquid-liquid extraction up to equilibrium and then centrifuged at (15557 g, $1 \mathrm{~h}$ ). The total concentration of 3-HP in the organic phase, as determined by back-extraction with $\mathrm{NaOH}$ solutions, ranged between 1 and $50 \mathrm{~g} / \mathrm{L}$. For the sake of band identification and to understand the mechanisms, other spectra were acquired, in particular for water, a commercial aqueous solution of 3-HP and organic phases after hydrochloric acid and 3-HP extraction.

\subsubsection{Reactive extractions}

Reactive liquid-liquid extractions of 3-hydroxypropionic acid in organic phases consisting of mixtures of purified tri-n-octylamine and $n$-decanol were performed. The initial concentration of 3-HP ranged between 0.25 and $50 \mathrm{~g} / \mathrm{L}$ in order to represent depleted bioconversion broth conditions. All experimental and analytical conditions were previously detailed [12]. Previously published 
experimental data [12] were used as a calibration set for the models. Another set of independent extractions at $25^{\circ} \mathrm{C}$ was performed in order to validate the predictive nature of the models in the concentration range tested. Initial conditions of both sets of experiments are given in Table 1.

Table 1: Experimental calibration and validation sets

\begin{tabular}{|c|c|c|c|c|c|}
\hline \multicolumn{4}{|c|}{ Calibration set $\left(25^{\circ} \mathrm{C}\right)$} & \multicolumn{2}{|c|}{ Validation set $\left(25^{\circ} \mathrm{C}\right)$} \\
\hline $\begin{array}{c}\text { Initial 3-HP } \\
\text { concentration } \\
(\mathrm{g} / \mathrm{L})\end{array}$ & $\begin{array}{c}\text { Initial TOA } \\
\text { concentration } \\
(\% \mathrm{v} / \mathrm{v})\end{array}$ & $\begin{array}{c}\text { Initial 3-HP } \\
\text { concentration } \\
(\mathrm{g} / \mathrm{L})\end{array}$ & $\begin{array}{c}\text { Initial TOA } \\
\text { concentration } \\
(\% \mathrm{v} / \mathrm{v})\end{array}$ & $\begin{array}{c}\text { Initial 3-HP } \\
\text { concentration } \\
(\mathrm{g} / \mathrm{L})\end{array}$ & $\begin{array}{c}\text { Initial TOA } \\
\text { concentration } \\
(\% \mathrm{v} / \mathrm{v})\end{array}$ \\
\hline 1 & 0 & 0.25 & 20 & 0.5 & 10 \\
\hline 1 & 10 & 0.5 & 20 & 2 & 30 \\
\hline 1 & 20 & 0.75 & 20 & 3.5 & 25 \\
\hline 1 & 30 & 2 & 20 & 5 & 15 \\
\hline 1 & 40 & 5 & 20 & 5 & 50 \\
\hline 1 & 50 & 7 & 20 & 15 & 40 \\
\hline 1 & 60 & 10 & 20 & 20 & 10 \\
\hline 1 & 70 & 20 & 20 & 30 & 40 \\
\hline 1 & 80 & 30 & 20 & 40 & 80 \\
\hline 1 & 90 & 40 & 20 & & \\
\hline 1 & 100 & 50 & 20 & & \\
\hline
\end{tabular}

Validation points were chosen in order to cover a large range of the experimental concentrations except the zone of low acid and high amine concentration, which is of little practical interest.

\subsubsection{Determination of model parameters}

As detailed above in the Theory section, the following model parameters had to be determined based on experimental data in order to predict the final composition of the system and, consequently, the extraction yield: $K_{11}, K_{11 n}, K_{c o m p}, K_{a q}, n$ and $p$ for Model 1 and $K_{11}\left(x_{R O H}^{i n i}\right)$, i.e., polynomial coefficients $a_{k}$ and $K_{a q}$, for Model 2. All calculations were performed with Matlab ${ }^{\circledR}$ 2015b numeric computation software (MathWorks, Natick, MA, USA) equipped with the Optimization and Statistics Toolboxes. The specified parameters were initialized to 1 and their values were iteratively refined, aiming at the best possible fit (in a least-squares sense) between the predicted and the measured extraction yields and equilibrium $\mathrm{pH}$, for the experiments in the calibration dataset. Model calibration based on two different types of experimental measurements simultaneously aims at reproducing real physical mechanisms at best. The acidic $\mathrm{pH}$ values for the fitting procedure have been multiplied by $1 / 7$ in order to have numeric values between 0 and 1 , in 
the same order of magnitude as the yield values. Yield and $\mathrm{pH}$ thus had a similar weight in the model calibration process, resulting in a better fit. Iterative parameter estimation was performed using the nlinfit function in Matlab, implementing the Levenberg-Marquardt optimization algorithm. For Model 1 , in each iteration of the algorithm and for each initial experimental condition, the system of equations (8) and (19-21) with bounds (11-13) and (21) was solved for the equilibrium concentrations $[R O H],\left[T O A_{\text {org }}\right],\left[A H_{a q}\right]$ and $\left[O A_{\text {org }}\right]$ using the Isqnonlin function of Matlab. For Model 2, in each iteration of the algorithm and for each initial experimental condition, the system of equations (6-8) with bounds (11-13) was solved for the equilibrium variables $\left[\right.$ TOA $\left.A_{\text {org }}\right],\left[A H_{a q}\right]$ and $\left[O A_{\text {org }}\right]$ using the Isqnonlin function of Matlab.

The Standard Error of the Regression for a variable $\mathrm{X}$ for both models was used to evaluate their accuracy, with $\mathrm{X}$ being either yield or $\mathrm{pH}$ :

$$
S E R=\sqrt{\frac{\sum_{i=1}^{N_{1}}\left(X_{\text {mod }, i}-X_{i}\right)^{2}}{N_{1}-P}}
$$

where $N_{1}$ is the number of experimental values in the calibration dataset and $\mathrm{P}$ the number of fitted parameters.

For the validation dataset, the root mean squared deviation of a variable $\mathrm{X}$ was used to compare model predictions to measured values:

$$
R M S D=\sqrt{\frac{\sum_{i=1}^{N_{2}}\left(X_{\text {mod }, i}-X_{i}\right)^{2}}{N_{2}}}
$$

where $N_{2}$ is the total number of experimental values in the validation dataset.

The absolute error between model and experimental values was also calculated as follows:

$$
\left|\Delta X_{i}\right|=\left|X_{\bmod , i}-X_{i}\right|
$$

And the mean absolute error: 


$$
\overline{|\Delta X|}=\frac{1}{n} \sum_{i=1}^{n}\left|\Delta X_{i}\right|
$$

\section{Results and discussion}

\subsection{Nature of the acid:amine complex}

\subsubsection{Spectroscopic study}

The infrared spectroscopy study was performed to explore the complex formation and to elucidate its structure. IR spectra are presented in Figs. 1 (A to D) and 3 for different solutions, with identification of representative bands. Spectra of pure compounds can be found in the Appendix.
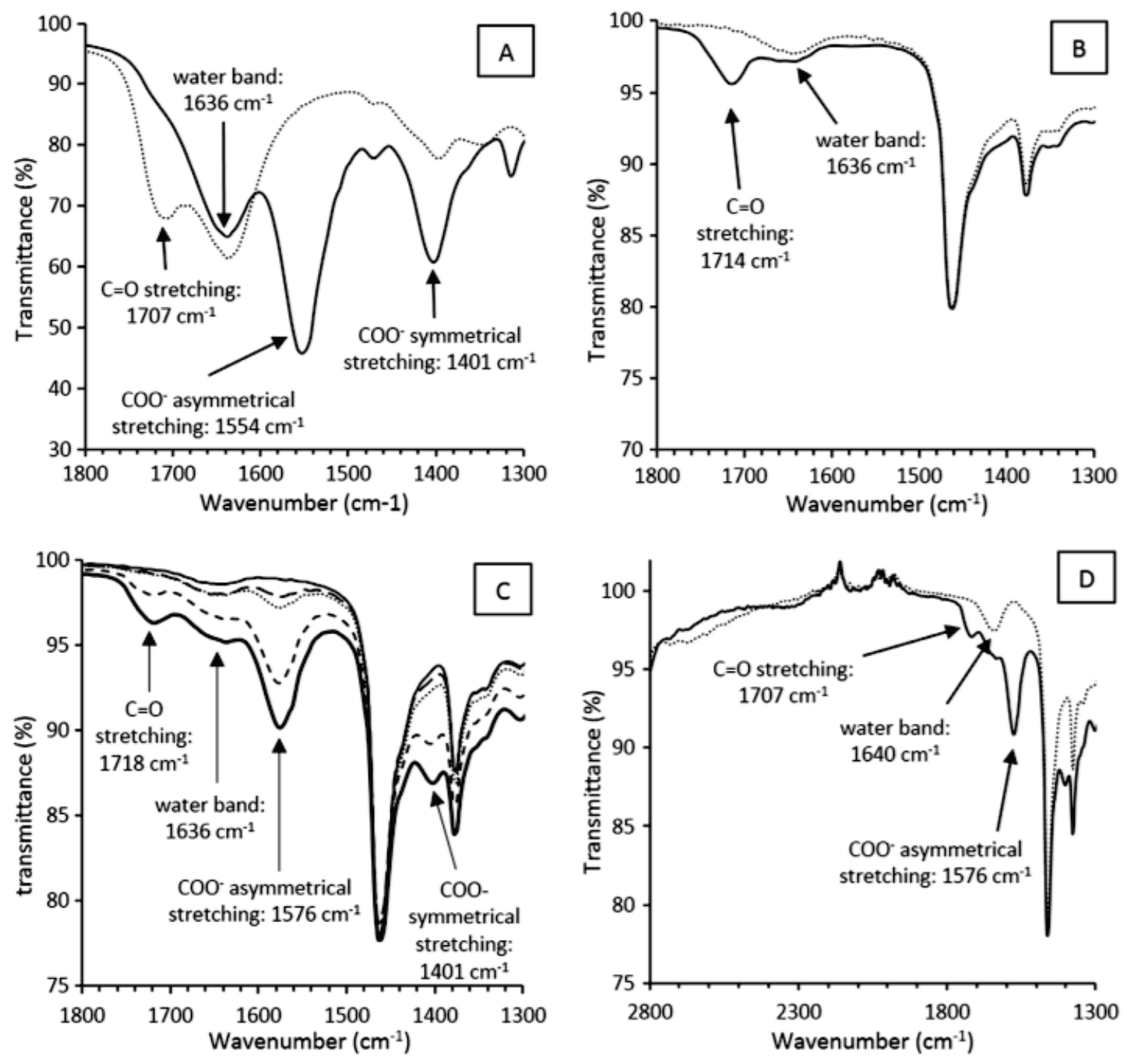
Figure 1: Infrared spectra:

- A: aqueous phase of the commercial 3-HP solution (dotted line) and the same one neutralized with $\mathrm{NaOH}$ (bold line);

- B: organic phases after physical extraction, i.e., using only decanol, of commercial 3-HP solution (bold line) and after contact with water alone (dotted line);

- C: organic phases (20\% V/V TOA in decanol) after reactive extractions of 3-HP (from upper spectrum: $0.8 \mathrm{~g} / \mathrm{L}, 4.5 \mathrm{~g} / \mathrm{L}, 7.7 \mathrm{~g} / \mathrm{L}, 33 \mathrm{~g} / \mathrm{L}$ and $53 \mathrm{~g} / \mathrm{L}$ of $3-H P$ in the organic phase);

- $\quad D$ : organic phases (20\% V/v TOA in decanol) after reactive extraction of $1.2 \mathrm{~mol} / \mathrm{L}$ of aqueous hydrochloric acid (dotted line) and $3-H P(53 \mathrm{~g} / \mathrm{L}$ in the organic phase).

It was observed that the main significant differences between spectra were situated in the 1300$1800 \mathrm{~cm}^{-1}$ region with six main bands (Fig. 1C), without solvent bands hindering variations between samples. That is why only this region is presented in most of the spectra. The band at $1636 \mathrm{~cm}^{-1} \mathrm{can}$ be attributed to water (see the water spectrum in the Appendix) extracted in the organic phase. Bands at 1463 and $1377 \mathrm{~cm}^{-1}$ are mainly due to the hydrocarbon skeletons of the organic species, as can be seen in decanol and TOA spectra in the Appendix, whereas the three other bands at 1401, 1575 , and $1718 \mathrm{~cm}^{-1}$ should be characteristic of chemical changes due to acid extraction.

According to the spectrum of the commercial solution in Fig. $1 \mathrm{~A}$, we can observe a band at $1707 \mathrm{~cm}^{-1}$ that is absent in water and in neutralized solution spectra. This band is attributed to the $C=0$ stretching of the hydrogen-bonded carbonyl function of the carboxyl group $(\mathrm{COOH})[30,31]$. In the neutralized solution spectra, two bands appear at 1403 and $1553 \mathrm{~cm}^{-1}$, which are attributed to symmetric and asymmetric stretching of the delocalized carboxylate group ( $\mathrm{COO}^{-}$), respectively $[30,31]$. These values are similar to other acids in aqueous phases [31]. Therefore, in Fig. $1 \mathrm{C}$, the bands at 1401 and $1575 \mathrm{~cm}^{-1}$ can be attributed to the carboxylate group and the band at $1718 \mathrm{~cm}^{-1}$ to the hydrogen-bonded carbonyl function of the carboxyl group. These values also correspond to other acids in organic phases containing amines [32].

The spectrum of the organic phase after extraction without amine (so-called physical extraction) (Fig. 1B) has a single characteristic band at $1714 \mathrm{~cm}^{-1}$. It can be attributed to $\mathrm{C}=\mathrm{O}$ stretching of the carboxyl group that is hydrogen-bonded [30,31] to decanol and/or other carboxyl groups (intermolecular) and/or to the alcohol function of 3-HP (intramolecular) and/or to water. It clearly 
shows that the behavior is totally different from the extraction with amines and that 3-HP only exists in its non-dissociated form.

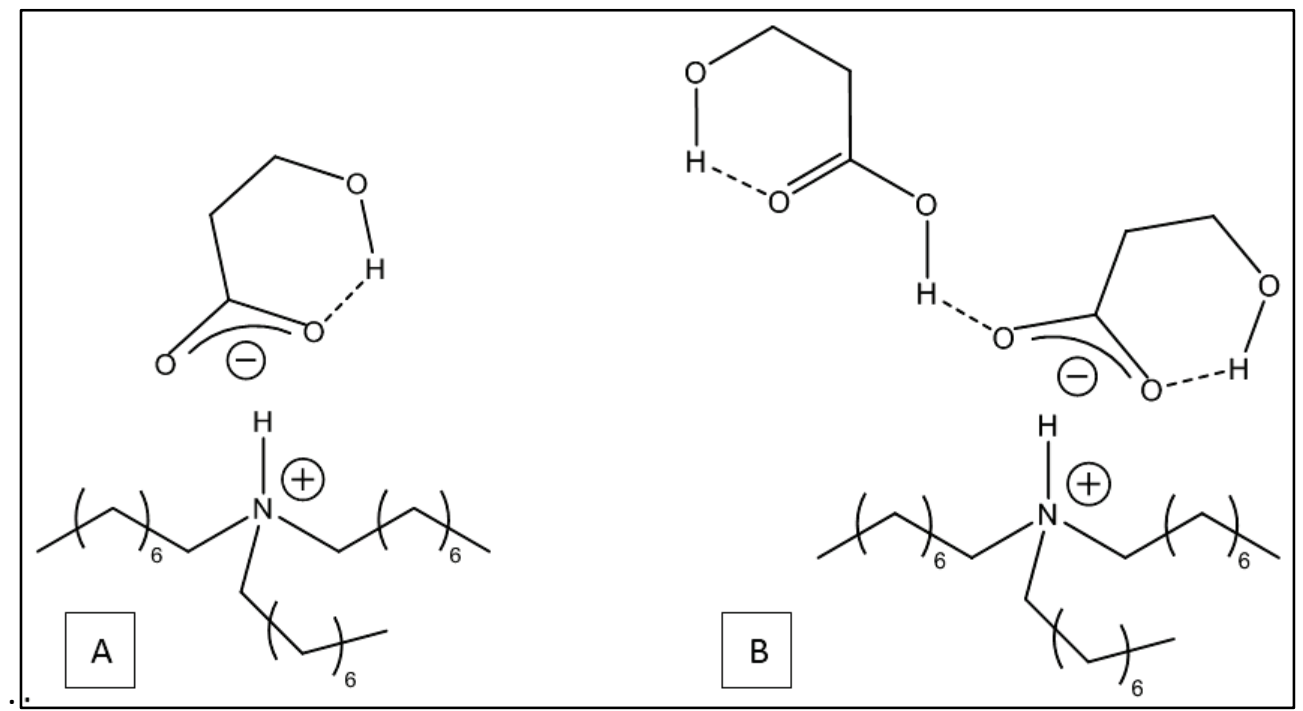

Figure 2: Proposed structures for the (1:1) (A) and (2:1) (B) acid-amine complexes.

In reactive extraction, the main band due to a carboxylate group $\left(1575 \mathrm{~cm}^{-1}\right)$ demonstrates strong interactions between the amine and the acidic proton of the carboxyl group. It can denote the dissociation of 3-HP in the organic phase, especially in an active proton-donating solvent like decanol and, hence, the formation of an ion pair as proposed in Fig. 2A, even though the expected very weak $\mathrm{N}-\mathrm{H}$ ammonium band is not obvious around $2500 \mathrm{~cm}^{-1}$. This absence is most probably due to the solvent (decanol + water) since even substantial hydrochloric acid extraction does not allow N-H band formation (Fig. 1D) and characteristic solvent bands hinder the observation of the specific bands of interest.

In order to further study the supposed $\mathrm{N}-\mathrm{H}$ bond, attempts were made to obtain the reaction products of acid:amine complexation in a concentrated form and to exclude the influence of the solvent. A comparison with a strong acid, hydrochloric acid, is also provided in order to have a better interpretation of spectra. The idea of the experiment is to force the separation of the organic phase into polar and apolar water insoluble phases. The polar phase appears after extraction between the aqueous and the top organic phase and is called the third phase, while the apolar phase remains at the top of the tube and includes the original organic phase compounds. The third phase is meant to 
consist mainly in acid:amine complexes, acid and water. When hydrochloric acid was used, a high initial concentration of $1.2 \mathrm{~mol} / \mathrm{L}$ in the aqueous phase was brought into contact with an organic phase made of $20 \% \mathrm{v} / \mathrm{v}$ TOA in $n$-decane. To observe the third phase formation in the case of 3-HP, it was necessary to directly use the commercial solution with pure TOA since $n$-decane totally prevented 3-HP extraction. The result was the formation of an extremely viscous third phase. Both third phases were recovered and their IR spectra are presented in Fig. 3.

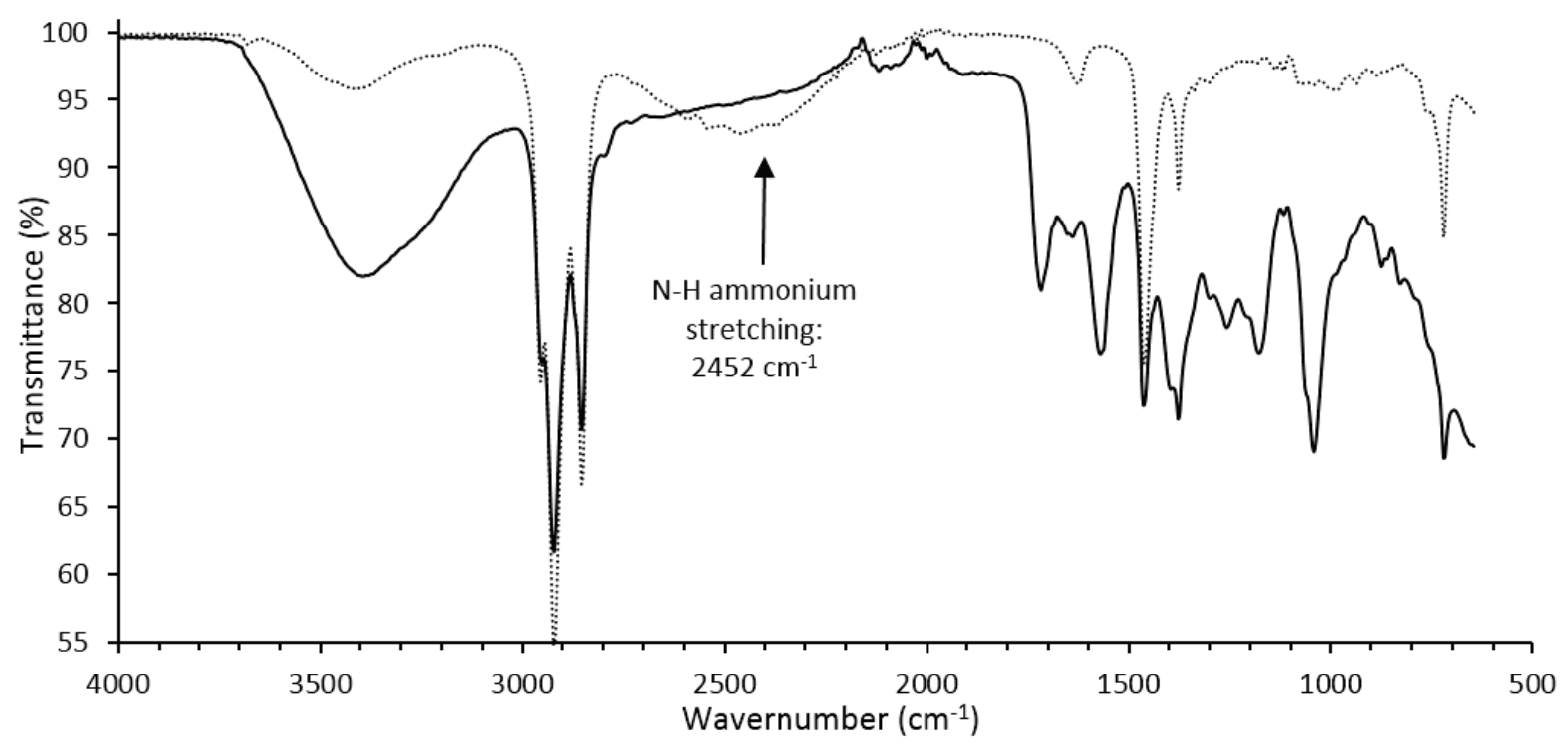

Figure 3: InfraRed spectra of third phases formed after extraction of $1.2 \mathrm{~mol} / \mathrm{L}$ of aqueous hydrochloric acid with 20\% V/V TOA in decane (dotted line) and commercial 3-HP solution with pure TOA (bold line)

With hydrochloric acid, a broad N-H band can be observed at $2500 \mathrm{~cm}^{-1}$ while the $\mathrm{C}-\mathrm{N}$ band at 1095 $\mathrm{cm}^{-1}$ and the Bohlmann band at $2795 \mathrm{~cm}^{-1}$ have disappeared (see TOA spectrum in the Appendix) indicating that the nitrogen lone pair is involved in a complex [33,34]. Contrary to hydrochloric acid and despite the observation of the carboxylate bands, the spectrum of the 3-HP third phase revealed no clear sign of the $\mathrm{N}-\mathrm{H}$ ammonium bond but, instead, a broad absorption covering the entire wavenumber range up to $3300 \mathrm{~cm}^{-1}$. This might be a sign of an unlocalized $\mathrm{N}-\mathrm{H}$ ammonium bond made impossible in the case of the strong acid [35] or simply a strong water/acid absorption. However, this situation is not very representative of the protic and quite polar decanol, which is a quite favorable medium for ionization. Results are not easily comparable as shown by the fact that ammonium bands are absent in decanol for both acids. 
In decanol, when the concentration of 3-HP is close to or exceeds the one of amine, another band appears at $1718 \mathrm{~cm}^{-1}$, corresponding to the carbonyl bond of the carboxyl group, like in Fig. $1 \mathrm{~B}$. Following are some reasons why this bond may be able to exist at such high concentrations of 3-HP:

(i) free acid is not negligible in solution as in the case of physical extraction (see Fig. 1B);

(ii) an equilibrium exists between ion-pairing and $\mathrm{H}$-bonding for acid:amine interactions shifted toward the ion pair;

(iii) more than one acid molecules is involved in complexes, supplementary molecules being $\mathrm{H}$ bonded to the first one, which is itself bonded to the ammonium part of the complex, as illustrated in Fig. 2B.

The third suggestion is the only one able to explain the overloading observed when comparing 3-HP $(53 \mathrm{~g} / \mathrm{L}=0.59 \mathrm{~mol} / \mathrm{L})$ and amine $(20 \% \mathrm{v} / \mathrm{v}=0.46 \mathrm{~mol} / \mathrm{L})$ concentrations at the highest tested value of acid concentration. This suggests the possibility to form (2:1) acid:amine complexes (Fig. 2B) for the highest concentrations tested in the calibration set. However, (2:1) acid:amine complexes should only concern a few concentrations in our experimental set and in weak proportions when compared to the (1:1) complex, so we will only consider the ion pair formation like in Fig. 2A.

\subsubsection{Validation of stoichiometry with model 0}

The stoichiometry of the acid-amine complex was first checked. For that, a simple model that only takes acid-amine complexes without solvation (Model 0) into account was tested for given excess amounts of TOA $(0.46 \mathrm{~mol} / \mathrm{L}=20 \% \mathrm{v} / \mathrm{v})$ and solvent, compared to the $3-\mathrm{HP}$ initial concentration $(0.011 \mathrm{~mol} / \mathrm{L}=1 \mathrm{~g} / \mathrm{L})$. The results are presented in Fig. 4 . 
Initial acid concentration (g/L)

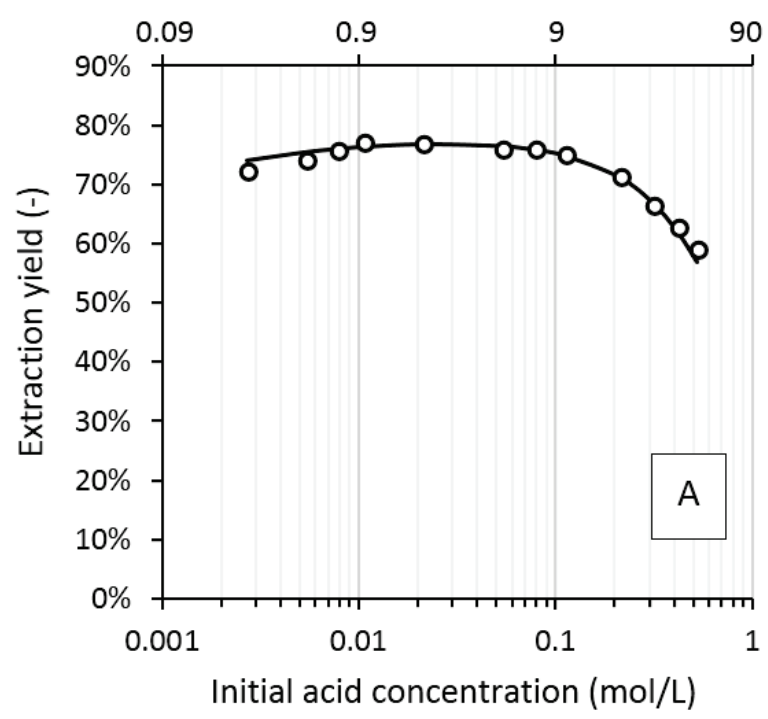

Initial acid concentration $(\mathrm{g} / \mathrm{L})$

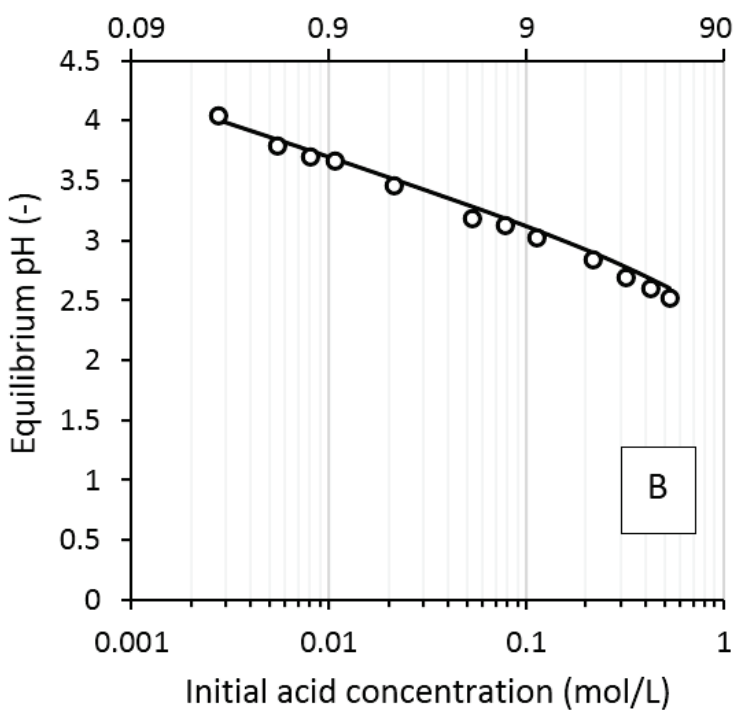

Figure 4: Experimental extraction yields $(A)$ and $\mathrm{pH}$ at equilibrium $(B)$ as a function of initial acid concentration (symbols). Calculation with Model 0 assuming a (1:1) stoichiometry for the

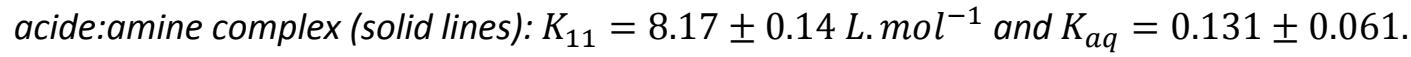

It appears that the (1:1) stoichiometry reasonably fits the data. This result aligns with the literature because the amine was in excess and the solvent is polar and protic, allowing intermolecular $\mathrm{H}$ bonding between the solvent and the complex, thus inhibiting acid oligomerization. This latter phenomenon is called overloading. Tamada and King [15] observed a lesser overloading of amines, i.e., $(n: 1)$ stoichiometry with $n>1$ when lactic acid was used compared to acetic acid. These authors speculated that this effect was due to the proximity between the hydroxyl group of lactic acid and the carboxylate group, allowing strained but possible intramolecular $\mathrm{H}$-bonding [30]. Indeed, theoretical calculations and experimental results proved the 5 -member ring $\mathrm{H}$-bondings as the most stable conformers for isolated lactic acid [36]. The 3-HP structure also shows strong interactions between its hydroxyl and carbonyl groups. The most stable conformations involve electrostatic interactions and $\mathrm{H}$ bonding between the $\mathrm{H}$ of the hydroxyl group and the carbonyl oxygen of the carboxyl group [37]. It results in the formation of less strained six-member rings (Fig. 2) and, consequently, is even more favorable than their 5-member counterparts in lactic acid. This conformation could be a part of the explanation for the observed (1:1) stoichiometry. 
We have therefore shown that Model 0 was able to provide accurate results for a given composition of the organic phase. Given the highly protic character of the solvent used, the possible intramolecular H-bonding involving the carboxyl part of the complex, the dilution of 3-HP and the excess of amines in the organic phase, we assume the (1:1) acid-amine stoichiometry to be the main complexation mechanism over the whole range tested. However, extrapolating the complexation equilibrium constant found to the calculation of equilibrium with other organic phase compositions (i.e., increasing the amine content and decreasing the solvent concentration) provided inaccurate predictions, as it can be seen in Fig. 5. This motivated the development of more complete models able to take the solvating power of the organic phase into account.
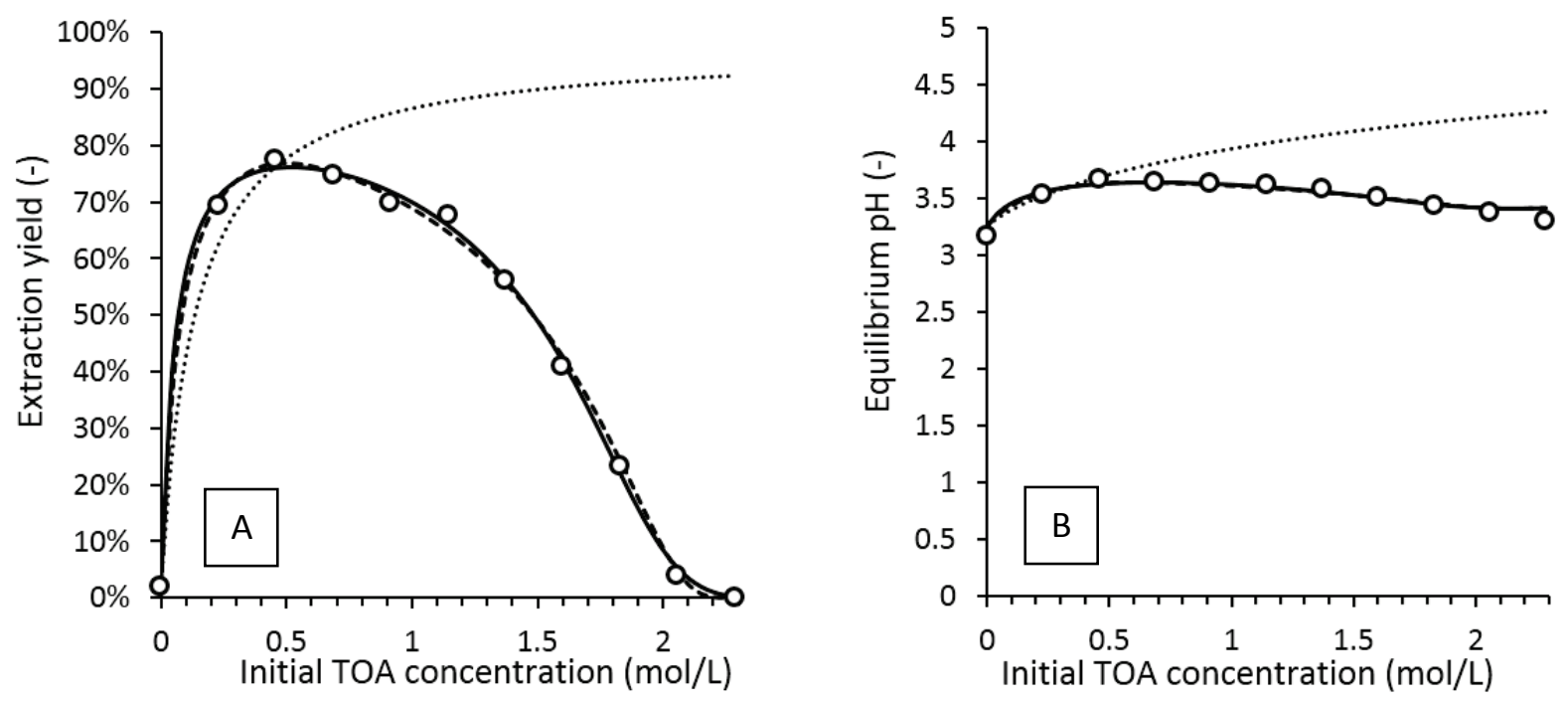

Figure 5: Experimental yields $(A)$ and $\mathrm{pH}(B)$ as a function of initial TOA concentration (symbols) with the calculations of Model $O$ (dotted lines), Model 1 (dashed lines) and Model 2 (solid lines).

\subsection{Calibration of Model 1}

Table 2: Parameters determined for Model 1

\begin{tabular}{|c|c|c|}
\cline { 2 - 3 } \multicolumn{1}{c|}{} & $\begin{array}{c}\text { Parameter } \\
\text { values }\end{array}$ & $\begin{array}{c}\text { Standard } \\
\text { error }\end{array}$ \\
\hline$K_{11}\left({\left.\mathrm{~L} . \mathrm{mol}^{-1}\right)}^{-(n+1)}\right)$ & 0 & - \\
\hline$K_{11 n}\left(\mathrm{~L}^{n+1} \cdot \mathrm{mol}^{-(n+1)}\right)$ & 0.88 & \pm 0.30 \\
\hline$n(-)$ & 4.63 & \pm 0.63 \\
\hline$K_{a q}(-)$ & 0.037 & \pm 0.015 \\
\hline$K_{\text {comp }}\left(L^{p+1} \cdot \mathrm{mol}^{-(p+1)}\right)$ & 0.162 & \pm 0.063 \\
\hline$p$ & 4.04 & \pm 0.77 \\
\hline \hline$R^{2}$ & \multicolumn{2}{|c}{0.996} \\
\hline
\end{tabular}


Parameters fitted by the numerical procedure and the coefficient of determination of the regression are given in Table 2. Parameter K11 was found to be not different from zero at a 0.05 significance level. It was therefore set to exactly 0 and the values of the other parameters recalculated accordingly. Figure 5 shows the results of Model 1 for the calibration data focusing on the influence of TOA concentration in the organic phase. Model 1 is able to reproduce experimental yield and $\mathrm{pH}$ quite accurately. Hence, a complex structure including the solvent is able to describe extraction yield decrease for high amine concentrations. The stoichiometry of decanol in the complex $(n)$ is found to be around 5. This solvation number allows the model to reproduce the bell-shaped profiles of the extraction yields. This type of model was used by Tamada and King [30] for succinic acid and Alamine 336 in chloroform. They found a stoichiometry of 2 for chloroform when used alone as a diluent and 3 when mixed with heptane, an inert diluent. The same was found when dichloromethane was used as the active diluent in a mixture with heptane. For malonic acid with Alamine 336 in MIBK, the solvent stoichiometry was found to be 2 . For synergistic extractants, solvation numbers of 1 and 2 for TBP and 1 or 2 for D2EHPA were found. Numerical values of extraction constants in this case are difficult to compare with the literature because they are not provided in the same units and the solvents used do not have the same molar volume, implying a great variability in molar concentrations. In a previous paper [12], we showed that each molecule of extracted 3-HP adds two molecules of water in the organic phase. Accordingly, a representation of the result of Model 1 would be the following complex: $\left[\left(\mathrm{TOAH}^{+}, \mathrm{A}^{-}\right)(\mathrm{ROH})_{5}\left(\mathrm{H}_{2} \mathrm{O}\right)_{2}\right]_{\text {org }}$. This is useful to predict the amount of water in the organic phase at equilibrium. However, such a high stoichiometric coefficient for decanol suggests a general solvation phenomenon described by non-stoichiometric interactions. This is the scope of Model 2. 

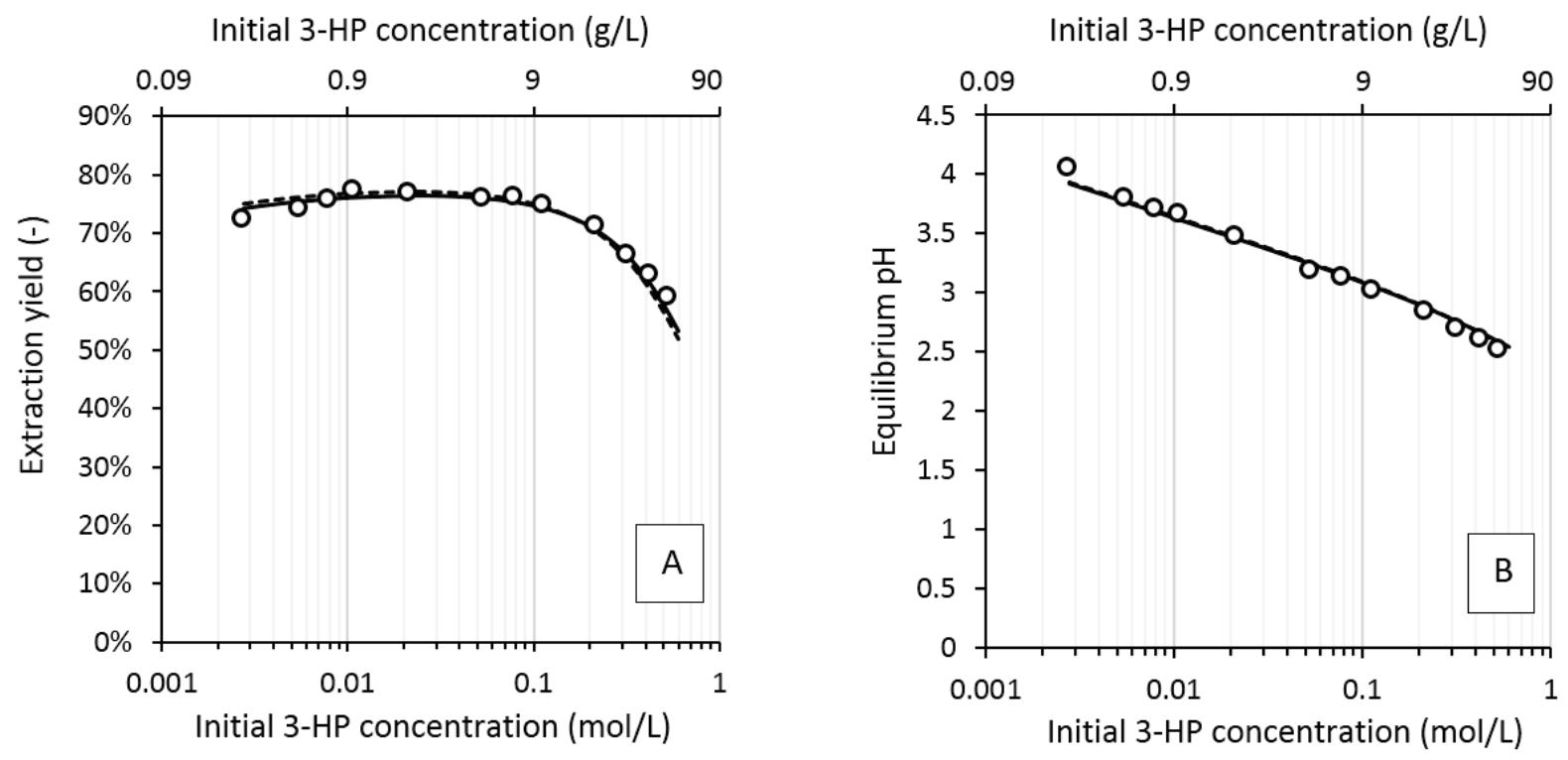

Figure 6: Experimental yields $(A)$ and $\mathrm{pH}(B)$ as a function of initial acid concentration (symbols) with simulations of Model 1 (dashed lines) and Model 2 (solid lines).

Figure 6 focuses on the influence of the initial acid concentration on the extraction yield and $\mathrm{pH}$.

Model values are in good agreement with measurements. The $\mathrm{pH}$ is correctly predicted by Model 1 in the entire range tested. The main parameter influencing the $\mathrm{pH}$ is $K_{a q}$, the transfer of impurities in the aqueous phase (reaction 5). We demonstrated in a previous paper [12] that this transfer was very weak in the case of purified TOA, with practically no effects. This is why a high uncertainty existed for the determined value of $K_{a q}$ (Table 2), suggesting that this parameter and reaction 5 could be removed from the model when using purified TOA.

\subsection{Calibration of Model 2}

Table 3: Parameter values determined for Model 2.

\begin{tabular}{|c|c|c|}
\cline { 2 - 3 } \multicolumn{1}{c|}{} & $\begin{array}{c}\text { Parameters } \\
\text { value }\end{array}$ & $\begin{array}{c}\text { Standard } \\
\text { error }\end{array}$ \\
\hline$a_{0}\left(\mathrm{~L} . ~ m o l^{-1}\right)$ & -6.84 & \pm 0.60 \\
\hline$a_{1}\left(\mathrm{~L} . ~ m o l^{-1}\right)$ & 19.7 & \pm 3.1 \\
\hline$a_{2}\left(\mathrm{~L} . ~ m o l^{-1}\right)$ & -19.4 & \pm 5.0 \\
\hline$a_{3}\left(\mathrm{~L} . \mathrm{mol}^{-1}\right)$ & 9.5 & \pm 2.6 \\
\hline$K_{a q}$ & 0.036 & \pm 0.012 \\
\hline \hline$R^{2}$ & \multicolumn{2}{|c|}{0.997} \\
\hline
\end{tabular}

The parameters determined for Model 2 and the corresponding coefficient of determination of the regression are given in Table 3. The polynomial order was found to be 3 . The relationship giving the 
value of the apparent equilibrium coefficient as a function of the solvent fraction (Equation 27) becomes:

$$
\ln \left(K_{11}\right) \approx-6.84+19.7 x_{R O H}^{i n i}-19.4\left(x_{R O H}^{i n i}\right)^{2}+9.5\left(x_{R O H}^{i n i}\right)^{3}
$$

It can be seen in Figs. 5 and 6 that Model 2 well fits the calibration data for the yields and pH.

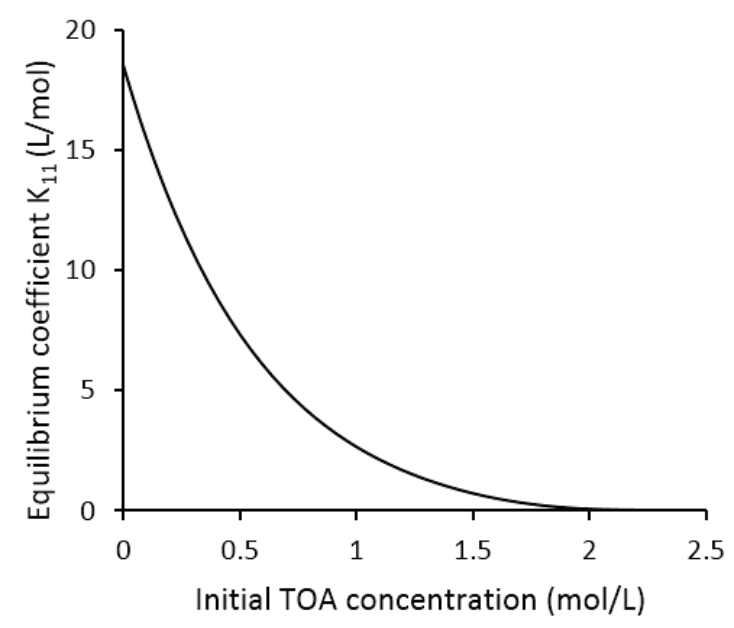

Figure 7: Apparent equilibrium coefficient of complexation as a function of the initial concentration of amine.

Figure 7 shows the variation of the apparent equilibrium coefficient with the initial amount of TOA in the organic phase. It appears that when the concentration of amine in the organic phase increases, this complexation coefficient decreases. In terms of activity coefficients, the equilibrium coefficient should be proportional to the TOA activity coefficient and inversely proportional to the complex activity coefficient. We know that the complex is insoluble in TOA, as shown by the formation of a third phase when using only TOA as an extractant phase. This proves that the complex is highly unstable in TOA, meaning that it should have a higher activity coefficient when the organic phase becomes enriched in TOA to the detriment of decanol, with better physicochemical properties. This is consistent with the fact that when the TOA concentration is too high, the solvation of the complex by decanol and water is less efficient. Since the TOA is essentially apolar and strictly $\mathrm{H}$-bond acceptor, it has no ability to stabilize the complex in the organic phase since the stabilization was shown to be due to the H-bond donor character of the solvent [12]. TOA is a hydrophobic species, insoluble in water, ethanol or acetone, for example, and should therefore be less stable in 
hydrophilic environments and more reactive, with a higher activity coefficient. Accordingly, this coefficient should decrease with the increase in the TOA content in alcohols. Indeed, we know from primary calculations (UNIFAC, not shown here) that its activity coefficient should decrease when increasing the amount of TOA from 10 to $20 \% \mathrm{v} / \mathrm{v}$ in a mixture containing decanol. All this explains the decrease of the equilibrium coefficient $K_{11}$ with the amount of TOA.

The values of the equilibrium coefficient $K_{11}$ determined in this study can be compared to similar extractions of other oxygenated C3 carboxylic acids. For example, Wasewar et al. [18] reported the complexation constant $K_{11}=45 \mathrm{~L}_{\mathrm{mol}} \mathrm{mol}^{-1}$ for lactic acid extraction in decanol by Alamine 336 in the range of $20-40 \% \mathrm{v} / \mathrm{v}(0.46-0.92 \mathrm{~mol} / \mathrm{L})$, and $\mathrm{Pal}$ et al. [19] reported the complexation constant $K_{11}=133{\mathrm{~L} . \mathrm{mol}^{-1}}^{-1}$ for pyruvic acid extraction in decanol by TOA in the range of $5-15 \% \mathrm{v} / \mathrm{v}(0.09-$ $0.28 \mathrm{~mol} / \mathrm{L})$. These higher values can be explained by the higher hydrophobicity and stronger acidity of these two acids when compared to 3-HP.

Equilibrium $\mathrm{pH}$ is also perfectly described by Model 2. The value of $K_{a q}$ is the same as in Model 1 , which is consistent with the fact that it is not meant to depend on the solvation model choice.

\subsection{Validation and comparison of Models 1 and 2}

Validation experiments involved initial concentrations other than those used for the calibration of the models. For predictions, model parameters were unchanged, as given in Tables 2 and 3. Figure 8 provides a visual comparison of model accuracy, while Table 4 gives the corresponding statistics. 

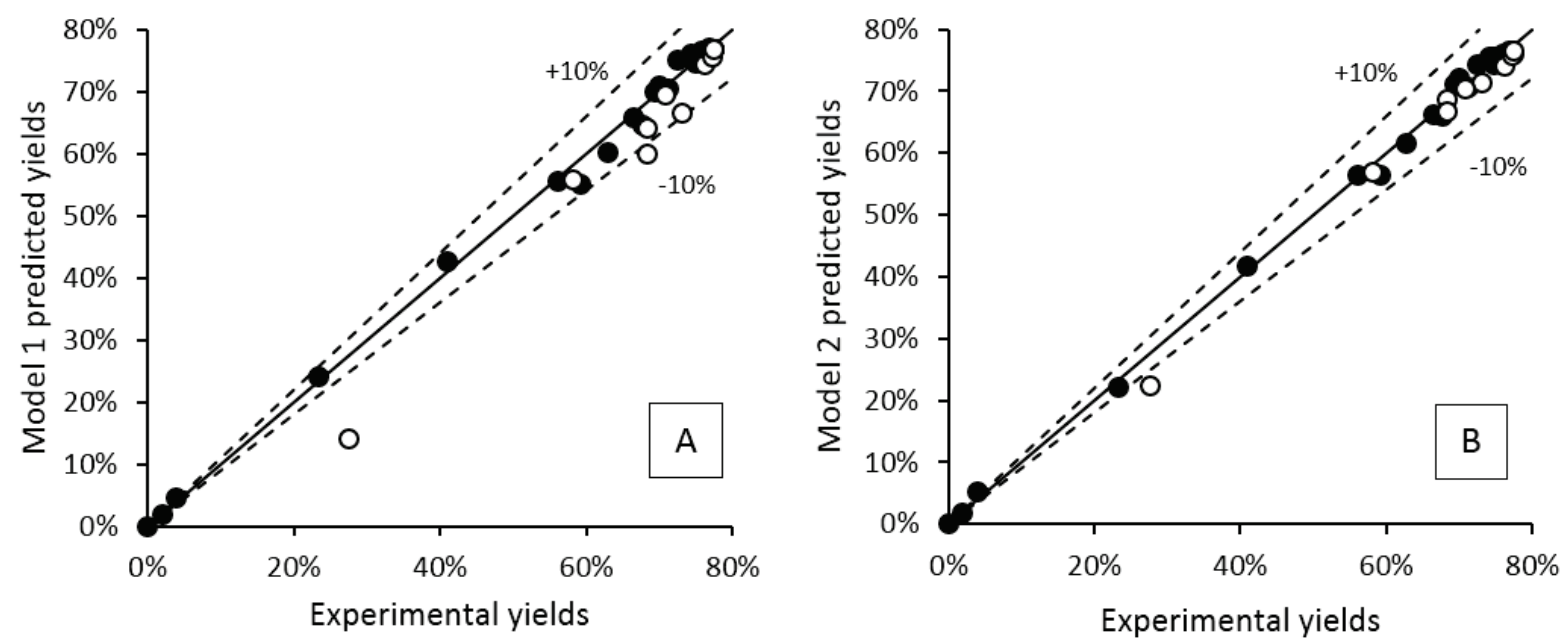

Figure 8: Model 1 (A) and Model 2 (B) predicted yields as a function of experimental yields for the calibration (filled circles) and the validation (open circles) experiments.

Table 4: Comparison of model accuracy, in percent of extraction yield

\begin{tabular}{cccccccc}
\hline & SER & $\overline{|\Delta Y|}$ & $\max (|\Delta Y|)$ & & $\operatorname{RMSD}$ & $\overline{|\Delta Y|}$ & $\max (|\Delta Y|)$ \\
\hline $\begin{array}{c}\text { Model 1 } \\
\text { calibration set }\end{array}$ & 1.7 & 1.1 & 4.2 & $\begin{array}{c}\text { Model 1 } \\
\text { validation set }\end{array}$ & 6.0 & 4.5 & 13.5 \\
\hline $\begin{array}{c}\text { Model 2 } \\
\text { calibration set }\end{array}$ & 1.4 & 1.0 & 2.6 & $\begin{array}{c}\text { Model 2 } \\
\text { validation set }\end{array}$ & 2.2 & 1.7 & 5.3 \\
\hline
\end{tabular}

It can be seen that both models provide good predictions with most of the points being within the $10 \%$ relative error. Calibrations of both models give very similar statistics. However, according to Table 4, Model 2 predictions appear to be two or three times more accurate than those of Model 1. The reason is to be found in the very structure of the models and this is emphasized when plotting the yield prediction surfaces of both models as a function of initial TOA and 3-HP concentrations (Fig. 9). It can be observed that surfaces present an optimal yield of $77 \%$, around $0.5 \mathrm{~mol} / \mathrm{L} \mathrm{TOA}(22 \% \mathrm{v} / \mathrm{v})$ and $0.024 \mathrm{~mol} / \mathrm{L} 3-\mathrm{HP}(2 \mathrm{~g} / \mathrm{L})$. 

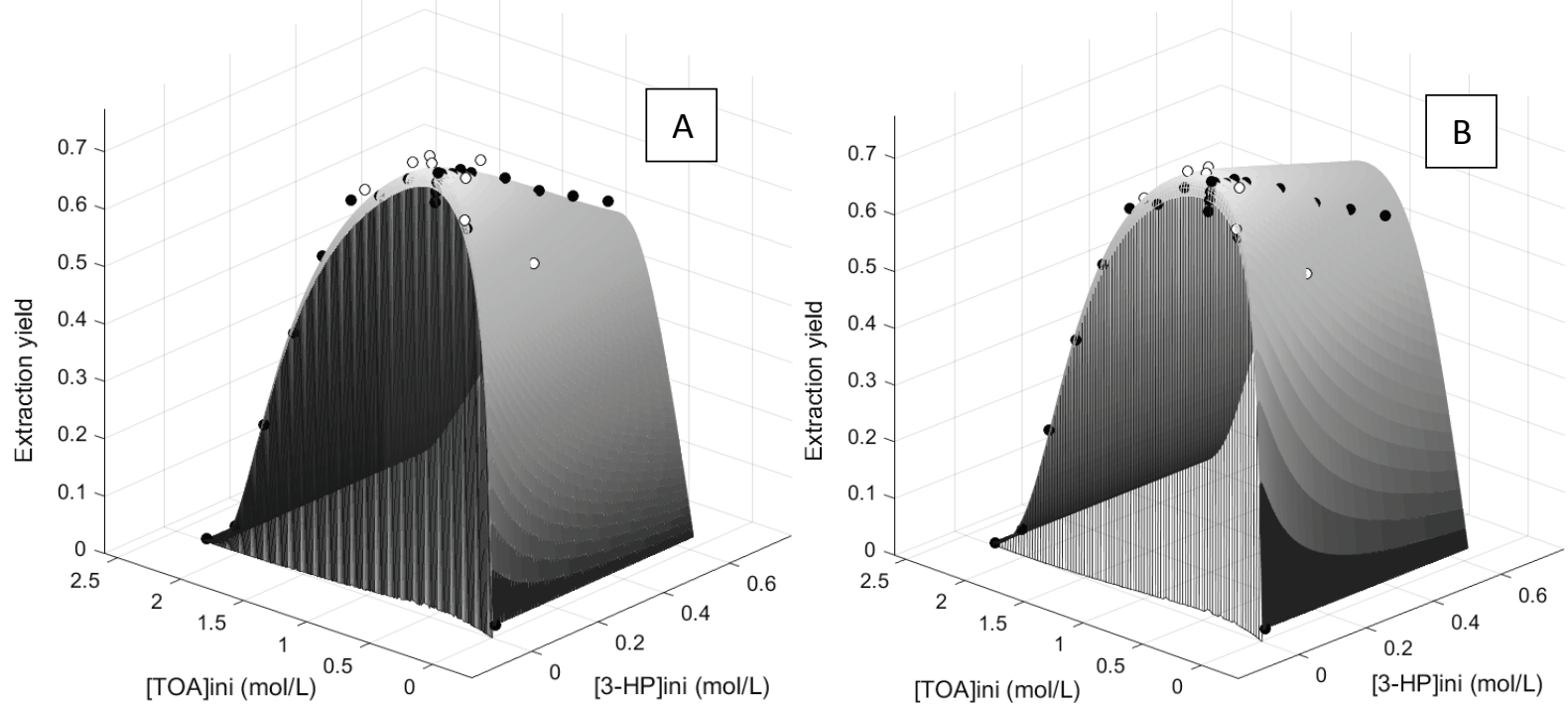

Figure 9: Extrapolation of Model 1 (A) and Model 2 (B) predictions in the whole range of acid and amine concentrations tested, with superimposed experimental measurements: calibration (filled circles) and validation (open circles).

It appears from Fig. 9 that the major discrepancy between both models is the faster decrease of the yield with the increase of initial acid concentration in Model 1 (left) than in Model 2 (right). This is due to the high consumption of decanol in the complex formation: nearly five molecules of decanol in one molecule of complex, implying that decanol is rapidly depleted, preventing the formation of even more complexes. This problem could certainly be avoided by adding more types of complexes corresponding to different degrees of solvation, but that would mean more parameters to fit a stoichiometric model with the possibility of non-stoichiometric phenomena. Moreover, this model totally ignores the importance of water in the acid extraction. In contrast, Model 2 with the same number of adjusted parameters representing global changes in the phase's properties provides better predictions and can therefore be seen as a better tool to describe reactive extraction.

Tamada $[9,30]$ warned that the stoichiometric model could only be used for strong interactions between the solvent and the acid-amine core. Indeed, solvation is a complex phenomenon resulting in non-stoichiometric organizations of mater in solutions [1], leading to non-ideal thermodynamic behavior. These assemblages may be the leading mechanism of the solvent effect [9]. Solvents like phosphoryl species are known to show stoichiometric behavior as extractants for complexation [1] 
and synergistic effects with amines [24], but they are capable of much stronger interactions than C-O solvents [38]. The higher polarity of the P-O bond $\left(\Delta \chi_{P-O}=1.25>\Delta \chi_{C-O}=0.89\right)$ and quantum considerations make the P-O bond an extremely polarized group in phosphine oxides, for example $[39,40]$. This explains the stronger Lewis base character of P-O extractants like TBP compared to C-O extractants, and the possibility to use defined stoichiometric coefficients over a large range of concentrations.

To sum up, the global decrease of the extraction yield with the increase of TOA concentration may be due to the reduction of the solvent available for $\mathrm{H}$-bonding to stabilize the complex, to an increase in competing interactions between decanol ( $\mathrm{H}$-bond donor) and TOA ( $\mathrm{H}$-bond acceptor), and to the decreased stabilization of the complex.

\section{Conclusion}

This paper describes an in-depth study of the acid:amine complexation when aqueous 3-HP is extracted by tri- $n$-octylamine in $n$-decanol. The main extraction mechanism for dilute aqueous solutions is the formation of an ion pair involving the carboxylate ion of 3-HP in the organic phase. This mechanism was described by a chemical model based on mass action laws but this usual model was shown to be unable to describe the impact of initial amine concentration in the organic phase (Model 0). To take the solvation effects of the diluting agent into account, i.e. decanol, the model had to describe yield variations, especially the sharp decrease in extraction yield when the decanol amount was low. Indeed, the variation of the physicochemical properties of the medium strongly shifts the complexation equilibrium because amine and solvent have very different properties $(\mathrm{H}-$ bond ability, polarity, dissociating power, etc.). This is better described by the variation of the complexation equilibrium constant (Model 2) rather than assuming the complexation of the solvent as a synergistic extractant on the acid:amine core (Model 1). The complexation equilibrium coefficient in Model 2 appeared to vary from 0 to $19{\mathrm{~L} . \mathrm{mol}^{-1}}^{-1}$ when the decanol proportion in the organic phase ranged between 0 and $100 \%$, denoting huge differences in system properties. This 
model allows a good prediction of extraction yield with only a $2 \%$ root mean squared deviation over wide concentration ranges (initially $0.25-50 \mathrm{~g} / \mathrm{L}$ aqueous $3-\mathrm{HP}$ and $0-100 \% \mathrm{v} / \mathrm{v}=0-2.3 \mathrm{~mol} / \mathrm{L} \mathrm{TOA}$ in the organic phase) and is potentially applicable to other reactive extraction systems.

\section{Acknowledgements}

The authors are grateful to the Champagne-Ardenne Region and the Conseil Départemental de la Marne and Reims Métropole for financial support. 
Appendices

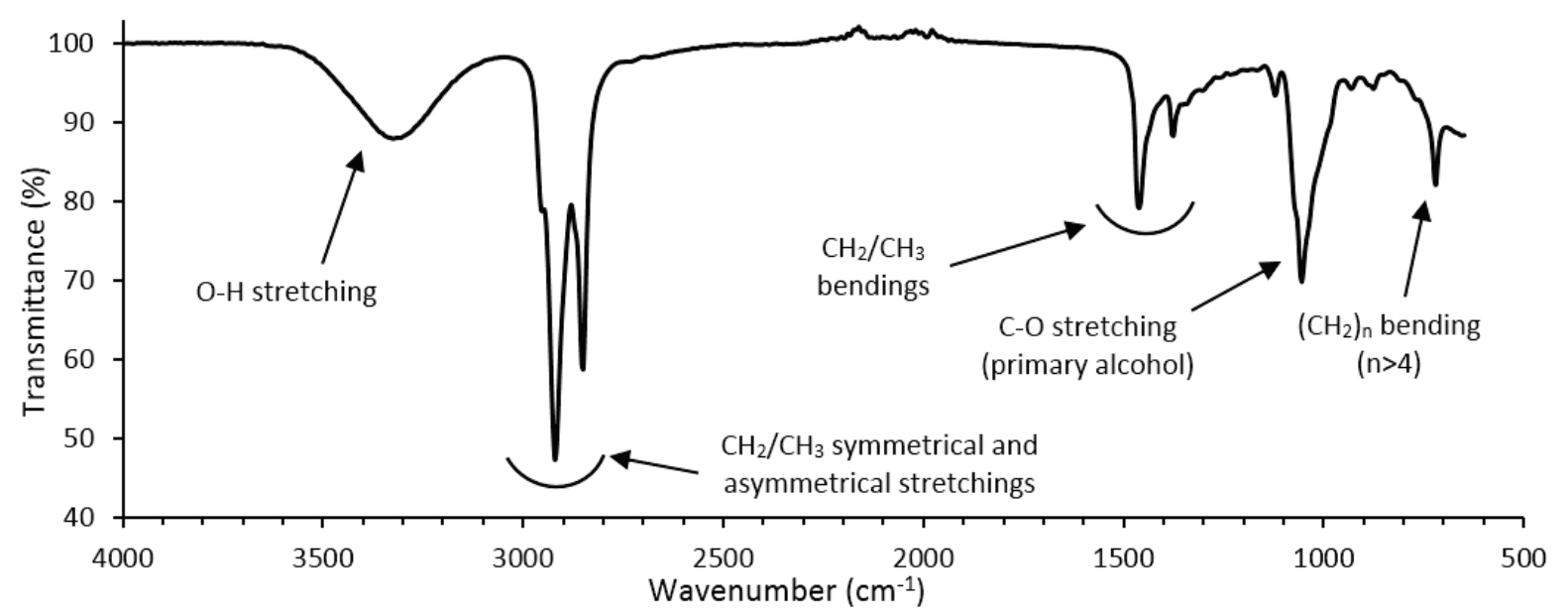

Appendix 1: Infrared spectrum of $n$-decanol.

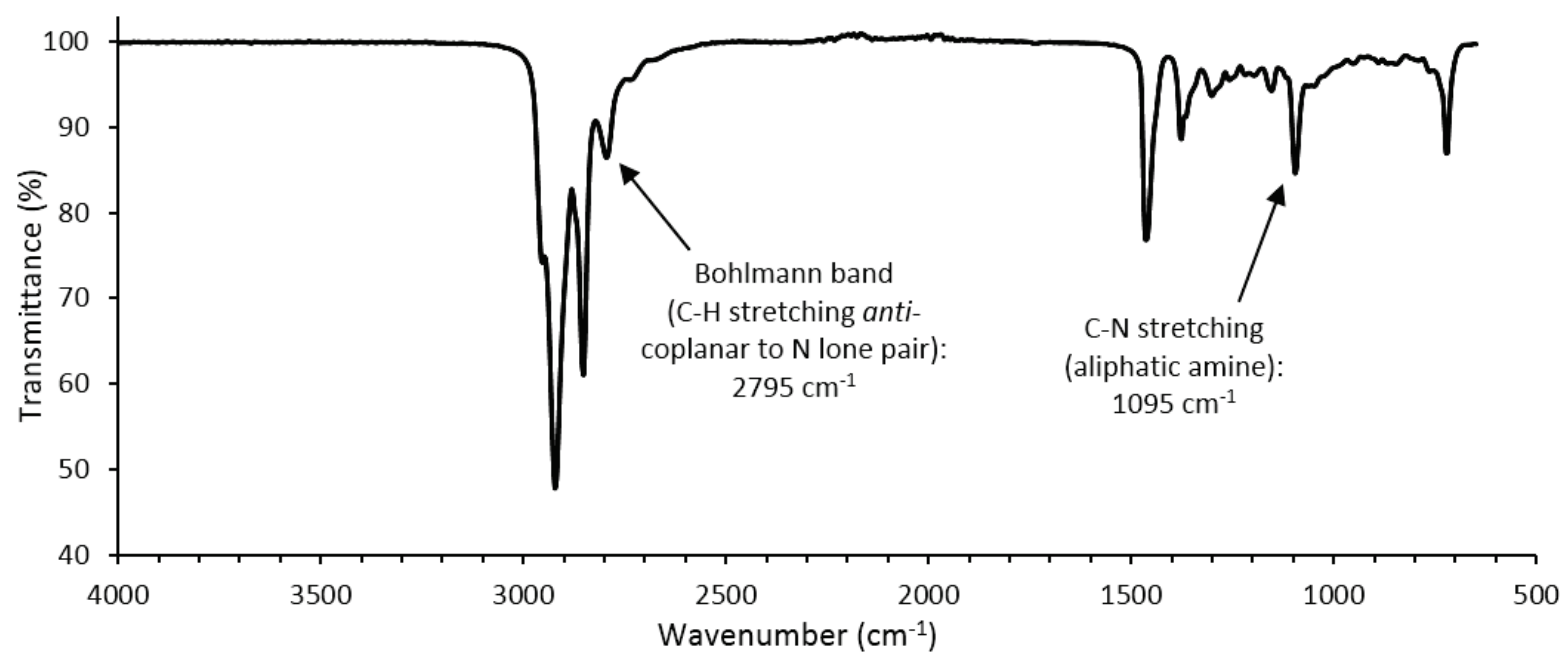

Appendix 2: Infrared spectrum of tri-n-octylamine.

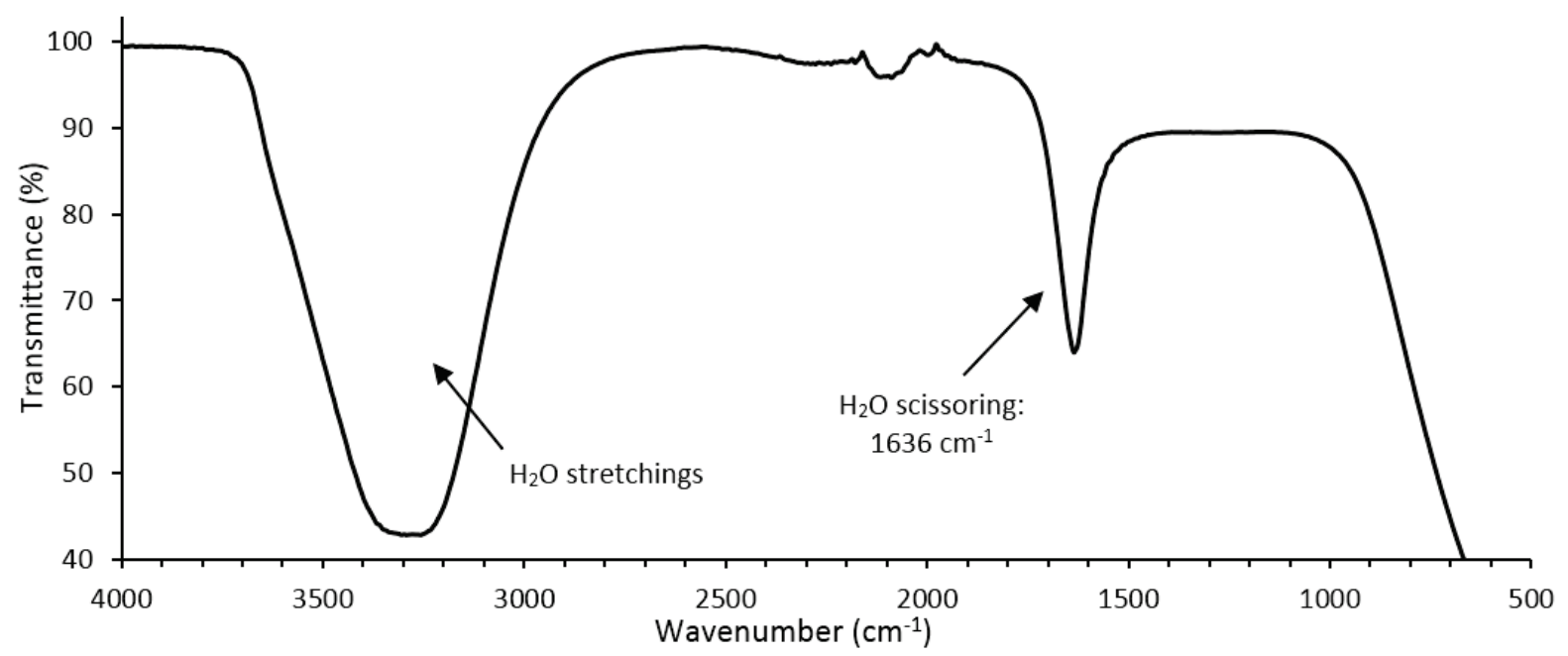

Appendix 3: Infrared spectrum of water. 
References

[1] A.S. Kertes, C.J. King, Extraction Chemistry of Fermentation Product Carboxylic Acids, Biotechnology and Bioengineering. 28 (1986) 269-282.

[2] V. Bízek, J. Horáček, M. Koušová, Amine extraction of citric acid: effect of diluent, Chemical Engineering Science. 48 (1993) 1447-1457.

[3] H. Uslu, C. Bayat, S. Gökmen, Y. Yorulmaz, Reactive extraction and LSER model consideration of lactic acid with tripropylamine+organic solvent systems from aqueous solution at room temperature, Desalination. 249 (2009) 694-698.

[4] N. Marchitan, C. Cojocaru, A. Mereuta, G. Duca, I. Cretescu, M. Gonta, Modeling and optimization of tartaric acid reactive extraction from aqueous solutions: A comparison between response surface methodology and artificial neural network, Separation and Purification Technology. 75 (2010) 273-285.

[5] D. Datta, S. Kumar, Modeling using response surface methodology and optimization using differential evolution of reactive extraction of glycolic acid, Chemical Engineering Communications. 202 (2015) 59-69.

[6] E.E. Spala, J.E. Ricker, Thermodynamic Model for Solvating Solutions with Physical Interactions, Industrial \& Engineering Chemistry Process Design and Development. 21 (1982) 409-415.

[7] T. Kirsch, H. Ziegenfuls s, G. Maurer, Distribution of citric, acetic and oxalic acids between water and organic solutions of tri-n-octylamine, Fluid Phase Equilibria. 129 (1997) 235-266.

[8] G. Maurer, Modeling the liquid-liquid equilibrium for the recovery of carboxylic acids from aqueous solutions, Fluid Phase Equilibria. 241 (2006) 86-95.

[9] J.A. Tamada, A.S. Kertes, C.J. King, Extraction of Carboxylic Acids with Amine Extractants. 1. Equilibria and Law of Mass Action Modeling, Industrial \& Engineering Chemistry Research. 29 (1990) 1319-1326.

[10] A. Keshav, K.L. Wasewar, S. Chand, Extraction of propionic acid with tri-n-octyl amine in different diluents, Separation and Purification Technology. 63 (2008) 179-183.

[11] H. Uslu, D. Datta, Experimental and Theoretical Investigations on the Reactive Extraction of Itaconic (2-Methylidenebutanedioic) Acid Using Trioctylamine ( $N, N$-Dioctyloctan-1-amine), Journal of Chemical \& Engineering Data. 60 (2015) 1426-1433.

[12] F. Chemarin, M. Moussa, M. Chadni, B. Pollet, P. Lieben, F. Allais, I.C. Trelea, V. Athès, New insights in reactive extraction mechanisms of organic acids: An experimental approach for 3hydroxypropionic acid extraction with tri-n-octylamine, Separation and Purification Technology. 179 (2017) 523-532.

[13] S. Kumar, B.V. Babu, K.L. Wasewar, Investigations of biocompatible systems for reactive extraction of propionic acid using aminic extractants (TOA and Aliquat 336), Biotechnology and Bioprocess Engineering. 17 (2012) 1252-1260.

[14] A. Keshav, K.L. Wasewar, S. Chand, REACTIVE EXTRACTION OF PROPIONIC ACID USING TRI-nOCTYLAMINE, Chemical Engineering Communications. 197 (2009) 606-626.

[15] A.F. Tuyun, H. Uslu, Reactive extraction of acrylic acid using trioctylamine (TOA) in versatile diluents, Desalination and Water Treatment. 55 (2015) 193-198.

[16] H. Uslu, Investigation of acrylic acid extractability from aqueous solution using tridodecyl amine extractant, Desalination and Water Treatment. 28 (2011) 189-195. doi:10.5004/dwt.2011.2246.

[17] D. Yankov, J. Molinier, J. Albet, G. Malmary, G. Kyuchoukov, Lactic acid extraction from aqueous solutions with tri-n-octylamine dissolved in decanol and dodecane, Biochemical Engineering Journal. 21 (2004) 63-71.

[18] K.L. Wasewar, A.B.M. Heesink, G.F. Versteeg, V.G. Pangarkar, Equilibria and kinetics for reactive extraction of lactic acid using Alamine 336 in decanol, Journal of Chemical Technology \& Biotechnology. 77 (2002) 1068-1075. 
[19] D. Pal, A. Tripathi, A. Shukla, K.R. Gupta, A. Keshav, Reactive Extraction of Pyruvic Acid Using Trin-octylamine Diluted in Decanol/Kerosene: Equilibrium and Effect of Temperature, Journal of Chemical \& Engineering Data. 60 (2015) 860-869.

[20] D. Pal, N. Thakre, A. Kumar, A. Keshav, Reactive extraction of pyruvic acid using mixed extractants, Separation Science and Technology. 51 (2016) 1141-1150.

[21] T. Werpy, G. Petersen, A. Aden, J. Bozell, J. Holladay, J. White, A. Manheim, D. Elliot, L. Lasure, S. Jones, M. Gerber, K. Ibsen, L. Lumberg, S. Kelley, Top Value Added Chemicals from Biomass, Volume 1-Results of Screening for Potential Candidates from Sugars and Synthesis Gas, U.S. Department of Energy, Oak Ridge, TN, 2004.

[22] A. Koudriavtsev, R.F. Jameson, W. Linert, The Law of Mass Action, Springer Berlin Heidelberg, Berlin, Heidelberg, 2001.

[23] A. Keshav, K.L. Wasewar, S. Chand, H. Uslu, Effect of binary extractants and modifier-diluents systems on equilbria of propionic acid extraction, Fluid Phase Equilibria. 275 (2009) 21-26.

[24] M. Matsumoto, T. Otono, K. Kondo, Synergistic extraction of organic acids with tri-n-octylamine and tri-n-butylphosphate, Separation and Purification Technology. 24 (2001) 337-342.

[25] R.-S. Juang, R.-T. Wu, Effect of a Water-Insoluble Organic Acid on Amine Extraction of Acetic Acid from Aqueous Solutions. Equilibrium Studies, Journal of Chemical Technology \& Biotechnology. 66 (1996) 160-168.

[26] M. Matsumoto, Y. Kawamura, K. Kondo, Synergistic Extraction of Organic Acids with Tri-nOctylamine and Di-2-Ethylhexylphosphoric Acid, Journal of Chemical Engineering of Japan. 37 (2004) 1150-1154.

[27] M. Moussa, G. Burgé, F. Chemarin, R. Bounader, C. Saulou-Bérion, F. Allais, H.-E. Spinnler, V. Athès, Reactive extraction of 3-hydroxypropionic acid from model aqueous solutions and real bioconversion media. Comparison with its isomer 2-hydroxypropionic (lactic) acid, J. Chem. Technol. Biotechnol. 91 (2015) 2276-2285.

[28] R.S. Hansen, F.A. Miller, S.D. Christian, Activity Coefficients of Components in the Systems Water-Acetic Acid, Water-Propionic Acid and Water-n-Butyric Acid at 25 $5^{\circ}$, The Journal of Physical Chemistry. 59 (1955) 391-395.

[29] C.W. Davies, Ion Association, Butterworths, London, 1962.

[30] J.A. Tamada, C.J. King, Extraction of Carboxylic Acids by Amines Extractant, Lawrence Berkeley Laboratory, CA (USA), United States, 1989.

[31] J.-J. Max, C. Chapados, Infrared Spectroscopy of Aqueous Carboxylic Acids: Comparison between Different Acids and Their Salts, The Journal of Physical Chemistry A. 108 (2004) 3324-3337.

[32] J.A. Tamada, C.J. King, Extraction of Carboxylic Acids with Amine Extractants. 2. Chemical Interactions and Interpretations of Data, Industrial \& Engineering Chemistry Research. 29 (1990) 1327-1333.

[33] J.A. Richman Jr, A qualitative study of the "Bohlmann Band," Master's Theses, Paper 986, University of Richmond, 1967.

[34] T. Kirsch, G. Maurer, Distribution of oxalic acid between water and organic solutions of tri-noctylamine, Industrial \& Engineering Chemistry Research. 35 (1996) 1722-1735.

[35] D.F. DeTar, R.W. Novak, Carboxylic acid-amine equilibria in nonaqueous solvents, Journal of the American Chemical Society. 92 (1970) 1361-1365.

[36] P. Nagy, Competing Intramolecular vs. Intermolecular Hydrogen Bonds in Solution, International Journal of Molecular Sciences. 15 (2014) 19562-19633.

[37] V.K.W. Cheng, M. Flock, M. Ramek, Ab Initio SCF Structure Investigation of $\beta$-Hydroxypropionic Acid and 3-Aminopropionamide, International Journal of Quantum Chemistry. 57 (1996) 929941.

[38] J.M. Wardell, C.J. King, Solvent equilibriums for extraction of carboxylic acids from water, Journal of Chemical and Engineering Data. 23 (1978) 144-148.

[39] D.B. Chesnut, A. Savin, The electron localization function (ELF) description of the PO bond in phosphine oxide, Journal of the American Chemical Society. 121 (1999) 2335-2336. 
[40] D.G. Gilheany, No d orbitals but Walsh diagrams and maybe banana bonds: chemical bonding in phosphines, phosphine oxides, and phosphonium ylides, Chemical Reviews. 94 (1994) 13391374. 